\title{
Identifying the space source term problem for a generalization of the fractional diffusion equation with hyper-Bessel operator
}

\author{
Nguyen Hoang Luc ${ }^{1}$, Le Nhat Huynh ${ }^{2,3}$, Dumitru Baleanu ${ }^{4,5,6}$ and Nguyen Huu Can ${ }^{7 *}$ (D)
}

\author{
"Correspondence: \\ nguyenhuucan@tdtu.edu.vn \\ ${ }^{7}$ Applied Analysis Research Group, \\ Faculty of Mathematics and \\ Statistics, Ton Duc Thang University, \\ Ho Chi Minh City, Vietnam \\ Full list of author information is \\ available at the end of the article
}

\begin{abstract}
In this paper, we consider an inverse problem of identifying the source term for a generalization of the time-fractional diffusion equation, where regularized hyper-Bessel operator is used instead of the time derivative. First, we investigate the existence of our source term; the conditional stability for the inverse source problem is also investigated. Then, we show that the backward problem is ill-posed; the fractional Landweber method and the fractional Tikhonov method are used to deal with this inverse problem, and the regularized solution is also obtained. We present convergence rates for the regularized solution to the exact solution by using an a priori regularization parameter choice rule and an a posteriori parameter choice rule. Finally, we present a numerical example to illustrate the proposed method.
\end{abstract}

Keywords: Source term; Time-fractional diffusion equation; Ill-posed problem; Hyper-Bessel operator

\section{Introduction}

Fractional calculus has a long history in the mathematical theory and has attracted much attention in various fields of the applied science $[3,4,13,23]$. Fractional differential equations have an important position in the mathematical modeling of different physical systems [1, 10, 21], in engineering [6, 18], [7], and finance [24], in physics, chemistry, medicine, and they describe anomalous diffusion $[12,16,20]$.

In this paper, we restore the space source term problem for a generalization of the timefractional diffusion equation with variable coefficients. The time-fractional diffusion is discussed in this paper as follows:

$$
\begin{cases}{ }^{C}\left(t^{1-\beta} \frac{\partial}{\partial t}\right)^{\alpha} \mathrm{u}(\mathrm{x}, t)-\mathscr{B} \mathrm{u}(\mathrm{x}, t)=\mathcal{F}(\mathrm{x}, t), & (\mathrm{x}, t) \in \Omega \times(0, T), \\ \mathrm{u}(\mathrm{x}, t)=0, & (\mathrm{x}, t) \in \partial \Omega \times(0, T), \\ \mathrm{u}(\mathrm{x}, 0)=\mathrm{g}(\mathrm{x}), & \mathrm{x} \in \Omega,\end{cases}
$$

where $\Omega$ is bounded with sufficient smooth boundary $\partial \Omega$ in $\mathbb{R}^{d}(d \in \mathbb{N}), T>0$ is a fixed value, $0<\beta<1$, and ${ }^{C}\left(t^{1-\beta} \frac{\partial}{\partial t}\right)^{\alpha}$ stands for a regularized Caputo-like counterpart hyper-

(c) The Author(s) 2020. This article is licensed under a Creative Commons Attribution 4.0 International License, which permits use, sharing, adaptation, distribution and reproduction in any medium or format, as long as you give appropriate credit to the original author(s) and the source, provide a link to the Creative Commons licence, and indicate if changes were made. The images or other third party material in this article are included in the article's Creative Commons licence, unless indicated otherwise in a credit line to the material. If material is not included in the article's Creative Commons licence and your intended use is not permitted by statutory regulation or exceeds the permitted use, you will need to obtain permission directly from the copyright holder. To view a copy of this licence, visit http://creativecommons.org/licenses/by/4.0/. 
Bessel operator of order $0<\alpha<1$. From [10], we have the following formula:

$$
C\left(t^{1-\beta} \frac{\partial}{\partial t}\right)^{\alpha} \mathrm{v}(t)=\left(t^{1-\beta} \frac{\partial}{\partial t}\right)^{\alpha} \mathrm{v}(t)-\frac{\mathrm{v}(0) t^{-\alpha \beta}}{\beta^{-\alpha} \Gamma(1-\alpha)}
$$

where $\Gamma(x)$ is a gamma function, and the hyper-Bessel operator $\left(t^{1-\beta} \frac{\partial}{\partial t}\right)^{\alpha}$ was introduced by Dimovski in [8]. Since (2), we see that the study of (1) comes from the definition of hyper-Bessel operator. Some papers $[10,11]$ used the hyper-Bessel operator to describe heat diffusion for fractional Brownian motion. Some more details on them can be found in $[2,25,30]$.

The results for equation (1) were investigated by some recent works [2, 29]. The authors [2] considered two direct and inverse source problems of a fractional diffusion equation with regularized Caputo-like counterpart hyper-Bessel operator. They established the existence and uniqueness of solutions to the problem and gave the explicit eigenfunction expansions. In [29], the author investigated the exact solution of the inhomogeneous linear equation and the semilinear equation using fixed point theorems. In practice, some initial data, boundary data, diffusion coefficients, or source terms may not be given. By adding some given data, we can recover them, this is the inverse problem (or backward problem) of the time-fractional diffusion. To the best of our knowledge, the source identification problem for the fractional diffusion equation with hyper-Bessel operator has also been studied very little.

Our purpose in this paper is to find an inversion source problem for (1). Assume that the source term $\mathcal{F}(\mathrm{x}, t)$ of problem (1) is a forward problem, which can be split into $\mathcal{F}(\mathrm{x}) \mathcal{Q}(t)$, where $\mathcal{Q}(t)$ is known in advance. Hence, we want to identify the space source term $\mathcal{F}(\mathrm{x})$ by using the value of the final time $T$ as follows:

$$
\mathrm{u}(\mathrm{x}, T)=\mathscr{H}(\mathrm{x}), \quad \mathrm{x} \in \Omega .
$$

In fact, the measurements are noised, the observation data $\mathscr{H}$ are obtained by inexact data using some measurements; and so, they are approximated data by $\mathscr{H}^{\varepsilon}$ and

$$
\left\|\mathscr{H}^{\varepsilon}-\mathcal{H}\right\|_{\mathcal{L}^{2}(\Omega)} \leq \varepsilon
$$

where $\varepsilon>0$ is a bound on the measurement error. A small error of the given observation $\mathscr{H}$ can result in that the solution may have a large error. Hence, we have to propose some regularization method in order to recover stable approximations for the unknown space source function.

In this paper, we apply the fractional Landweber method and fractional Tikhonov method to restore the unknown space source function $\mathcal{F}$. Both methods were studied by Klann and Ramlau [15] when they considered a linear ill-posed problem. Since the a priori bound of the exact solution cannot be known exactly in practice, we need to give a posteriori choice of the regularization parameter. Study for choosing the regularization parameter by the a priori rule is easier than that by a posteriori rule.

The paper is organized as follows. In Sect. 2, we recall some preliminary results. The exact solution, the ill-posedness of the inverse problem, and the conditional stability are also discussed in Sect. 2. In Sects. 3 and 4, we present the fractional Landweber regularization 
method and the fractional Tikhonov regularization method. The convergence estimate under an a priori assumption for the exact solution and the a posteriori regularization parameter choice rule are considered in there. In the last section, we present a numerical example to illustrate the proposed method.

\section{Identifying the space source term problem}

\subsection{Preliminary results}

In this section, we recall some useful results.

Let us consider the operator $\mathscr{B}$ on the domain $\mathcal{D}(-\mathscr{B}):=\mathcal{H}_{0}^{1}(\Omega) \cap \mathcal{H}^{2}(\Omega)$, and assume that $-\mathscr{B}$ has eigenvalues $a_{p}$ with corresponding eigenfunction $w_{p} \in \mathcal{D}(-\mathscr{B})$.

Note

$$
0<a_{1} \leq a_{2} \leq a_{3} \leq \cdots \leq a_{p} \leq \cdots
$$

and $a_{p} \rightarrow \infty$ as $p \rightarrow \infty$. The most popular example of $\mathcal{B}$ is the negative Laplacian operator $-\Delta$ on $\mathcal{L}^{2}(\Omega)$, we have

$$
\begin{cases}\mathcal{B} w_{p}(\mathrm{x})=-a_{p} w_{p}(\mathrm{x}), & \text { for } \mathrm{x} \in \Omega, \\ w_{p}(\mathrm{x})=0, & \text { for } \mathrm{x} \in \partial \Omega\end{cases}
$$

From [5], it easy to see that $a_{p} \geq C p^{\frac{2}{d}}$ for $C$ is a constant, $p \in \mathbb{N}$, and $d$ is the dimensional number of the spatial variable.

Now, let us define fractional powers of $\mathscr{B}$ and the Hilbert scale spaces. For all $k \geq 0$, we denote by $(-\mathscr{B})^{k}$ the following operator:

$$
\mathcal{B}^{k} v:= \begin{cases}\sum_{p=1}^{\infty} a_{p}^{k}\left\langle v, w_{p}\right\rangle w_{p}, & \text { if } k \neq 0, \\ \sum_{p=1}^{\infty}\left\langle v, w_{p}\right\rangle w_{p}, & \text { if } k=0,\end{cases}
$$

and

$$
v \in \mathcal{D}\left((-\mathcal{B})^{k}\right):=\left\{v \in \mathcal{L}^{2}(\Omega): \sum_{p=1}^{\infty} a_{p}^{2 k}\left|\left\langle v, w_{p}\right\rangle\right|^{2}<\infty\right\} .
$$

The space $\mathcal{D}\left((-\mathscr{B})^{k}\right)$ is a Banach space with the following norm:

$$
\|x\|_{\mathcal{D}\left((-\mathcal{B})^{k}\right)}:=\left(\sum_{p=1}^{\infty} a_{p}^{2 k}\left|\left\langle x, w_{p}\right\rangle\right|^{2}\right)^{\frac{1}{2}}, \quad x \in \mathcal{D}\left((-\mathscr{B})^{k}\right) .
$$

It is easy to see that $\|v\|_{\mathcal{D}\left((-\mathscr{B})^{k}\right)}=\left\|(-\mathscr{B})^{k} v\right\|_{\mathcal{L}^{2}(\Omega)}$. Its domain $\mathcal{D}\left((-\mathscr{B})^{-k}\right)$ is a Hilbert space endowed with the dual inner product $\langle\cdot, \cdot\rangle_{-k, k}$ taking between $\mathcal{D}\left((-\mathscr{B})^{-k}\right)$ and $\mathcal{D}\left((-\mathscr{B})^{k}\right)$. This generates the norm

$$
\|v\|_{\mathcal{D}\left((-\mathcal{B})^{-k}\right)}=\left(\sum_{p=1}^{\infty} a_{p}^{-2 k}\left|\left\langle v, w_{p}\right\rangle_{-k, k}\right|^{2}\right)^{\frac{1}{2}} .
$$


Definition 2.1 ([21]) The generalized Mittag-Leffler function is defined as follows:

$$
E_{\alpha, \beta}(z)=\sum_{p=0}^{\infty} \frac{z^{p}}{\Gamma(\alpha p+\beta)}, \quad z \in \mathbb{C}, \text { for } \alpha>0, \beta \in \mathbb{R} .
$$

Note $E_{\alpha, \beta}(z)$ is an entire function in $z \in \mathbb{C}$. For convenience, let us set $E_{\alpha}(z):=E_{\alpha, 1}(z)$ and $\boldsymbol{E}(z):=E_{\alpha, \alpha}(z)$.

Lemma 2.1 (see [21]) Let $0<\alpha<2$, and $\beta \in \mathbb{R}$ be arbitrary. Let us suppose that $\mu$ is such that $\frac{\pi \alpha}{2}<\mu<\min \{\pi, \pi \alpha\}$. Then there exists a constant $\mathbb{M}=\mathbb{M}(\alpha, \beta, \mu)>0$ such that

$$
E_{\alpha, \beta}(-z) \leq \frac{\mathbb{M}}{1+|z|} ; \quad \mu \leq|\arg (z)| \leq \pi
$$

Lemma 2.2 (see $[21,26])$ Let $\alpha \in(0,1)$, then $E_{\alpha}(-z)>0$ for any $z>0$. Moreover, there exist three positive constants $\mathbb{M}_{\alpha, \beta}^{-}, \mathbb{M}_{\alpha, \beta}^{+}, \mathbb{M}_{\alpha, \beta}$ such that

$$
\frac{\mathbb{M}_{\alpha, \beta}^{-}}{1+z} \leq E_{\alpha}(-z) \leq \frac{\mathbb{M}_{\alpha, \beta}^{+}}{1+z}, \quad \boldsymbol{E}(-z) \leq \frac{\mathbb{M}_{\alpha, \beta}}{1+z}
$$

If $\alpha \in\left[\alpha_{0}, \alpha_{1}\right]$ for any $0<\alpha_{0}<\alpha_{1}<1$, the constants can be chosen, which depends only $\alpha_{0}, \alpha_{1}$.

Lemma 2.3 (see [21]) Let $\mathrm{c}>0$ and $0<\alpha<1$. Then

(a) $\frac{d}{d t} E_{\alpha}\left(-c t^{\alpha}\right)=-c t^{\alpha-1} \boldsymbol{E}\left(-c t^{\alpha}\right), t>0$;

(b) $\frac{d}{d t}\left(t^{\alpha-1} \boldsymbol{E}\left(-c t^{\alpha}\right)\right)=t^{\alpha-2} E_{\alpha, \alpha-1}\left(-c t^{\alpha}\right), t>0$;

(c) $\partial_{t}^{\alpha} E_{\alpha}\left(-\mathrm{c} t^{\alpha}\right)=-\mathrm{c} E_{\alpha}\left(-\mathrm{c} t^{\alpha}\right), t>0$;

(d) $\partial_{t}^{\alpha}\left(t^{\alpha-1} \boldsymbol{E}\left(-c t^{\alpha}\right)\right)=-c t^{\alpha-1} \boldsymbol{E}\left(-c t^{\alpha}\right), t>0$.

Lemma 2.4 ([17, 27]) For $0<\mathrm{k}<1, \mathrm{q}>0$, and $\mathrm{m} \in \mathbb{N}$, we obtain

$$
(1-k)^{m} k^{q} \leq q^{q}(m+1)^{-q}<q^{q} m^{-q} .
$$

Lemma 2.5 (see [28]) For some positive constants $r, \mu, c, d$, we obtain

$$
\frac{\mu c^{2-r}}{\mu c^{2}+d} \leq \begin{cases}M_{1} \mu^{\frac{r}{2}}, & 0<r<2, \\ M_{2} \mu, & r \geq 2\end{cases}
$$

and

$$
\frac{\mu c^{1-r}}{\mu c^{2}+d} \leq \begin{cases}M_{3} \mu^{\frac{1+r}{2}}, & 0<r<1 \\ M_{4} \mu, & r \geq 1\end{cases}
$$

where $M_{1}=M_{1}(r, d)>0, M_{2}=M_{2}(r, d)>0, M_{3}=M_{3}(r, d)>0, M_{4}=M_{4}(r, d)>0$ are independent of $c$. 


\subsection{Solution for a fractional diffusion equation with regularized Caputo-like counterpart of a hyper-Bessel differential operator}

Using the Fourier series expansion and the properties of Mittag-Leffler, the exact solution of problem (1) is given by the following form (see [2, 29]):

$$
\begin{aligned}
\mathrm{u}(\mathrm{x}, t)= & \sum_{p=1}^{\infty}\left[E_{\alpha}\left(-\frac{a_{p}}{\beta^{\alpha}} t^{\alpha \beta}\right) \mathrm{g}_{p}\right. \\
& \left.+\frac{1}{\beta^{\alpha}} \int_{0}^{t}\left(t^{\beta}-\tau^{\beta}\right)^{\alpha-1} \boldsymbol{E}\left(-\frac{a_{p}}{\beta^{\alpha}}\left(t^{\beta}-\tau^{\beta}\right)^{\alpha}\right) \mathcal{F}_{p} \mathcal{Q}(\tau) d\left(\tau^{\beta}\right)\right] w_{p}(\mathrm{x}),
\end{aligned}
$$

where $\mathrm{g}_{p}=\left\langle\mathrm{g}, w_{p}\right\rangle, F_{p}(\tau)=\mathcal{Q}(\tau)\left\langle\mathcal{F}, w_{p}\right\rangle$ stands for its Fourier coefficient.

Let $t=T$ in, and we obtain

$$
\begin{aligned}
\mathscr{H}_{p}= & E_{\alpha}\left(-\frac{a_{p}}{\beta^{\alpha}} T^{\alpha \beta}\right) \mathrm{g}_{p} \\
& +\frac{1}{\beta^{\alpha}} \mathcal{F}_{p} \int_{0}^{T}\left(T^{\beta}-\tau^{\beta}\right)^{\alpha-1} \boldsymbol{E}\left(-\frac{a_{p}}{\beta^{\alpha}}\left(T^{\beta}-\tau^{\beta}\right)^{\alpha}\right) \mathcal{Q}(\tau) d\left(\tau^{\beta}\right),
\end{aligned}
$$

where $\mathscr{H}_{p}=\left\langle\mathcal{H}, w_{p}\right\rangle$ with $p \in \mathbb{N}, p \geq 1$.

Lemma 2.6 Let $\mathcal{Q}:[0, T] \rightarrow \mathbb{R}$ be a positive continuous function such that $\inf _{t \in[0, T]}|\mathcal{Q}(t)|=$ $\mathcal{Q}_{0}$. Assume that $\|\mathcal{Q}\|_{\infty}=\sup _{t \in[0, T]}|\mathcal{Q}(t)|$, then we get

$$
\begin{aligned}
\frac{1}{a_{p}} \mathcal{Q}_{0} \mathcal{M} & \leq \frac{1}{\beta^{\alpha}} \int_{0}^{T}\left(T^{\beta}-\tau^{\beta}\right)^{\alpha-1} \boldsymbol{E}\left(-\frac{a_{p}}{\beta^{\alpha}}\left(T^{\beta}-\tau^{\beta}\right)^{\alpha}\right) \mathcal{Q}(\tau) d\left(\tau^{\beta}\right) \\
& \leq \frac{1}{a_{p}} \overline{\mathbb{M}_{\alpha, \beta}^{+}}\|\mathcal{Q}\|_{\infty}
\end{aligned}
$$

for all $p \in \mathbb{N}$.

Proof First, by Lemma (2.2), we obtain

$$
\begin{aligned}
& \frac{1}{\beta^{\alpha}} \int_{0}^{T}\left(T^{\beta}-\tau^{\beta}\right)^{\alpha-1} \boldsymbol{E}\left(-\frac{a_{p}}{\beta^{\alpha}}\left(T^{\beta}-\tau^{\beta}\right)^{\alpha}\right) \mathcal{Q}(\tau) d\left(\tau^{\beta}\right) \\
& \quad \leq \frac{1}{\beta^{\alpha}}\|\mathcal{Q}\|_{\infty} \mathbb{M}_{\alpha, \beta}^{+} \int_{0}^{T} \frac{\left(T^{\beta}-\tau^{\beta}\right)^{\alpha-1}}{1+\frac{a_{p}}{\beta^{\alpha}}\left(T^{\beta}-\tau^{\beta}\right)^{\alpha}} d\left(\tau^{\beta}\right) \\
& \quad \leq \frac{1}{a_{p}} \overline{\mathbb{M}_{\alpha, \beta}^{+}}\|\mathcal{Q}\|_{\infty}
\end{aligned}
$$

where $\overline{\mathbb{M}_{\alpha, \beta}^{+}}:=M_{2}\left(\frac{2}{\alpha}, \frac{\beta^{\alpha}}{a_{p}}\right)$ by applying Lemma 2.5 for $r=\frac{2}{\alpha}$ and $d=\frac{\beta^{\alpha}}{a_{p}}$.

Otherwise, we also get

$$
\frac{1}{\beta^{\alpha}} \int_{0}^{T}\left(T^{\beta}-\tau^{\beta}\right)^{\alpha-1} \boldsymbol{E}\left(-\frac{a_{p}}{\beta^{\alpha}}\left(T^{\beta}-\tau^{\beta}\right)^{\alpha}\right) \mathcal{Q}(\tau) d\left(\tau^{\beta}\right) \geq \mathcal{Q}_{0} \frac{1}{a_{p}} \mathcal{M}
$$

where $\mathcal{M}=\left(1-E_{\alpha}\left(-\frac{a_{p}}{\beta^{\alpha}} T^{\alpha \beta}\right)\right)$. 


\subsection{III-posedness and stability estimates}

For any $\mathcal{H} \in \mathcal{L}^{2}(\Omega)$, let $\mathcal{K}: \mathcal{L}^{2}(\Omega) \rightarrow \mathcal{L}^{2}(\Omega)$ be the following operator:

$$
\begin{aligned}
(\mathcal{K H})(\mathrm{x}) & :=\int_{\Omega} \mu(\zeta, \mathrm{x}) \mathcal{H}(\zeta) d \zeta \\
& =\sum_{p=0}^{\infty} \frac{1}{\beta^{\alpha}} \int_{0}^{T}\left(T^{\beta}-\tau^{\beta}\right)^{\alpha-1} \boldsymbol{E}\left(-\frac{a_{p}}{\beta^{\alpha}}\left(T^{\beta}-\tau^{\beta}\right)^{\alpha}\right) \mathcal{Q}(\tau) d\left(\tau^{\beta}\right)\left\langle\mathcal{H}, w_{p}\right\rangle w_{p},
\end{aligned}
$$

where the kernel $\mu(\cdot, \cdot)$ is

$$
\mu(\mathrm{x}, \zeta):=\frac{1}{\beta^{\alpha}} \sum_{p=1}^{\infty} \int_{0}^{T}\left(T^{\beta}-\tau^{\beta}\right)^{\alpha-1} \boldsymbol{E}\left(-\frac{a_{p}}{\beta^{\alpha}}\left(T^{\beta}-\tau^{\beta}\right)^{\alpha}\right) \mathcal{Q}(\tau) d\left(\tau^{\beta}\right) w_{p}(\zeta) w_{p}(\mathrm{x}) .
$$

Since (8), our problem is of finding $\mathcal{F}$ which can be transformed into

$$
\mathcal{K} \mathcal{F}=\Theta,
$$

where

$$
\Theta(\mathrm{x}):=\sum_{p=1}^{\infty} \Theta_{p} w_{p}(\mathrm{x}), \quad \text { with } \Theta_{p}:=\mathscr{H}_{p}-E_{\alpha}\left(-\frac{a_{p}}{\beta^{\alpha}} T^{\alpha \beta}\right) \mathrm{g}_{p}
$$

Hence, we obtain

$$
\mathcal{F}_{p}=\frac{\Theta_{p}}{\frac{1}{\beta^{\alpha}} \int_{0}^{T}\left(T^{\beta}-\tau^{\beta}\right)^{\alpha-1} \boldsymbol{E}\left(-\frac{a_{p}}{\beta^{\alpha}}\left(T^{\beta}-\tau^{\beta}\right)^{\alpha}\right) \mathcal{Q}(\tau) d\left(\tau^{\beta}\right)} .
$$

It is easy to see that $\mathcal{K}: \mathcal{L}^{2}(\Omega) \rightarrow \mathcal{L}^{2}(\Omega)$ is a compact operator, then problem (10) is illposed. To give the ill-posedness problem, we propose an illustrative example. Assume that $\mathrm{g}=0$, we choose the final data $\mathscr{H}^{l}(\mathrm{x})=\frac{w_{l}(\mathrm{x})}{\sqrt{a_{l}}}$, then the corresponding source terms

$$
\begin{aligned}
\mathcal{F}^{l}(\mathrm{x}) & =\sum_{p=1}^{\infty} \frac{\mathscr{H}_{p}^{l}}{\frac{1}{\beta^{\alpha}} \int_{0}^{T}\left(T^{\beta}-\tau^{\beta}\right)^{\alpha-1} \boldsymbol{E}\left(-\frac{a_{p}}{\beta^{\alpha}}\left(T^{\beta}-\tau^{\beta}\right)^{\alpha}\right) \mathcal{Q}(\tau) d\left(\tau^{\beta}\right)} w_{p}(\mathrm{x}) \\
& =\frac{w_{l}(\mathrm{x})}{\frac{1}{\beta^{\alpha}} \sqrt{a_{l}} \int_{0}^{T}\left(T^{\beta}-\tau^{\beta}\right)^{\alpha-1} \boldsymbol{E}\left(-\frac{a_{p}}{\beta^{\alpha}}\left(T^{\beta}-\tau^{\beta}\right)^{\alpha}\right) \mathcal{Q}(\tau) d\left(\tau^{\beta}\right)} .
\end{aligned}
$$

By Lemma (2.6), we get $\left\|\mathcal{F}^{l}(\mathrm{x})\right\|_{\mathcal{L}^{2}(\Omega)} \geq \frac{\sqrt{a_{l}}}{\overline{\mathbb{M}_{\alpha, \beta}^{+}\|\mathcal{Q}\|_{\infty}}}$, hence $\lim _{l \rightarrow \infty}\left\|\mathcal{F}^{l}(\mathrm{x})\right\|_{\mathcal{L}^{2}(\Omega)} \rightarrow \infty$. But $\left\|\mathscr{H}^{l}\right\|_{\mathcal{L}^{2}(\Omega)}=\frac{1}{\sqrt{a_{p}}}$, or $\lim _{l \rightarrow \infty}\left\|\mathscr{H}^{l}(\mathrm{x})\right\|_{\mathcal{L}^{2}(\Omega)} \rightarrow 0$. Some of the above observations imply that our problem (1) satisfying (3) is ill-posed in the sense of Hadamard.

Next, we get conditional stability in the following theorem.

Theorem 2.1 Let $\mathcal{Q}:[0, T] \rightarrow \mathbb{R}$ for all $t \in[0, T]$. Assume that $\mathcal{P}$ is a positive constant and

$$
\|\mathcal{F}\|_{\mathcal{D}\left((-\mathscr{B})^{-\kappa}\right)} \leq \mathcal{P} \quad \text { for some } \kappa>0
$$


Then we obtain

$$
\|\mathcal{F}\|_{\mathcal{L}^{2}(\Omega)} \leq\left(\mathcal{Q}_{0} \mathcal{M}\right)^{-\frac{\kappa}{\kappa+1}}\|\Theta\|_{\mathcal{L}^{2}(\Omega)}^{\frac{\kappa}{\kappa+1}} \mathcal{P} \frac{1}{\kappa+1}
$$

Proof By Hölder's inequality and (11), we obtain

$$
\begin{aligned}
\| \mathcal{F} & \|_{\mathcal{L}^{2}(\Omega)}^{2} \\
& =\sum_{p=1}^{\infty} \frac{\Theta_{p}^{2}}{\left(\frac{1}{\beta^{\alpha}} \int_{0}^{T}\left(T^{\beta}-\tau^{\beta}\right)^{\alpha-1} \boldsymbol{E}\left(-\frac{a_{p}}{\beta^{\alpha}}\left(T^{\beta}-\tau^{\beta}\right)^{\alpha}\right) \mathcal{Q}(\tau) d\left(\tau^{\beta}\right)\right)^{2}} \\
& \leq\left[\sum_{p=1}^{\infty} \Theta_{p}^{2}\right]^{\frac{\kappa}{\kappa+1}}\left[\sum_{p=1}^{\infty} \frac{\Theta_{p}^{2}}{\left(\frac{1}{\beta^{\alpha}} \int_{0}^{T}\left(T^{\beta}-\tau^{\beta}\right)^{\alpha-1} \boldsymbol{E}\left(-\frac{a_{p}}{\beta^{\alpha}}\left(T^{\beta}-\tau^{\beta}\right)^{\alpha}\right) \mathcal{Q}(\tau) d\left(\tau^{\beta}\right)\right)^{2(\kappa+1)}}\right]^{\frac{1}{\kappa+1}} .
\end{aligned}
$$

Applying Lemma 2.6 and (11), we get

$$
\sum_{p=1}^{\infty} \frac{\Theta_{p}^{2}}{\left(\frac{1}{\beta^{\alpha}} \int_{0}^{T}\left(T^{\beta}-\tau^{\beta}\right)^{\alpha-1} \boldsymbol{E}\left(-\frac{a_{p}}{\beta^{\alpha}}\left(T^{\beta}-\tau^{\beta}\right)^{\alpha}\right) \mathcal{Q}(\tau) d\left(\tau^{\beta}\right)\right)^{2(\kappa+1)}} \leq \sum_{p=1}^{\infty} \frac{a_{p}^{2 \kappa} \mathcal{F}_{p}^{2}}{\mathcal{Q}_{0}^{2 \kappa} \mathcal{M}^{2 \kappa}} .
$$

Hence,

$$
\|\mathcal{F}\|_{\mathcal{L}^{2}(\Omega)} \leq\left(\mathcal{Q}_{0} \mathcal{M}\right)^{-\frac{\kappa}{\kappa+1}}\|\Theta\|_{\mathcal{L}^{2}(\Omega)}^{\frac{\kappa}{\kappa+1}} \mathcal{P}^{\frac{1}{\kappa+1}}
$$

We finish the proof.

\section{Fractional Landweber regularization method and convergence rate}

From Sect. 2, we know that problem (1) satisfying (3) is ill-posed. Therefore, we need a regularization method. Now, we propose a fractional Landweber regularization method to solve the ill-posed problem (1) satisfying (3). The convergence rates for the regularized solution under two parameter choice rules are also considered.

From [14], $\mathcal{K} \mathcal{F}=\Theta$ is equivalent to

$$
\mathcal{F}=\left(I-c \mathcal{K}^{*} \mathcal{K}\right) \mathcal{F}+c \mathcal{K}^{*} \Theta \quad \text { for any } c>0
$$

where $0<c<\|\mathcal{K}\|^{-2}$ and $\mathcal{K}^{*}$ is the adjoint operator of $\mathcal{K}$.

Applying the fractional Landweber method given by [15], we propose the following regularized solution with exact data $\mathscr{H}$ :

$$
\begin{aligned}
& \mathcal{F}_{\mathrm{m}, \theta}(\mathrm{x}) \\
& \quad=\sum_{p=1}^{\infty} \frac{\left[1-\left(1-c\left[\frac{1}{\beta^{\alpha}} \int_{0}^{T}\left(T^{\beta}-\tau^{\beta}\right)^{\alpha-1} \boldsymbol{E}\left(-\frac{a_{p}}{\beta^{\alpha}}\left(T^{\beta}-\tau^{\beta}\right)^{\alpha}\right) \mathcal{Q}(\tau) d\left(\tau^{\beta}\right)\right]^{2}\right)^{\mathrm{m}}\right]^{\theta}}{\frac{1}{\beta^{\alpha}} \int_{0}^{T}\left(T^{\beta}-\tau^{\beta}\right)^{\alpha-1} \boldsymbol{E}\left(-\frac{a_{p}}{\beta^{\alpha}}\left(T^{\beta}-\tau^{\beta}\right)^{\alpha}\right) \mathcal{Q}(\tau) d\left(\tau^{\beta}\right)}\left\langle\Theta, w_{p}\right\rangle w_{p} .
\end{aligned}
$$

If the observation data $\mathscr{H}$ is noised by $\mathcal{H}^{\varepsilon}$, then we have

$$
\begin{aligned}
& \mathcal{F}_{\mathrm{m}, \theta}^{\varepsilon}(\mathrm{x}) \\
& \quad=\sum_{p=1}^{\infty} \frac{\left[1-\left(1-c\left[\frac{1}{\beta^{\alpha}} \int_{0}^{T}\left(T^{\beta}-\tau^{\beta}\right)^{\alpha-1} \boldsymbol{E}\left(-\frac{a_{p}}{\beta^{\alpha}}\left(T^{\beta}-\tau^{\beta}\right)^{\alpha}\right) \mathcal{Q}(\tau) d\left(\tau^{\beta}\right)\right]^{2}\right)^{\mathrm{m}}\right]^{\theta}}{\frac{1}{\beta^{\alpha}} \int_{0}^{T}\left(T^{\beta}-\tau^{\beta}\right)^{\alpha-1} \boldsymbol{E}\left(-\frac{a_{p}}{\beta^{\alpha}}\left(T^{\beta}-\tau^{\beta}\right)^{\alpha}\right) \mathcal{Q}(\tau) d\left(\tau^{\beta}\right)}\left\langle\Theta^{\varepsilon}, w_{p}\right\rangle w_{p},
\end{aligned}
$$


here $\Theta^{\varepsilon}:=\mathscr{H}^{\varepsilon}-\sum_{p=1}^{\infty}\left(E_{\alpha, 1}\left(-\frac{a_{p}}{\beta^{\alpha}} T^{\alpha \beta}\right) \mathrm{g}_{p}\right) w_{p}(\mathrm{x}), \theta \in\left(\frac{1}{2}, 1\right]$ is the fractional order, and $\mathrm{m}>0$ is the iterative step and is a regularization parameter. Here, we note that when $\theta=1$, the fractional Landweber method becomes a standard Landweber regularization.

Lemma 3.1 Let $a_{p}>0, \theta \in\left(\frac{1}{2}, 1\right], \mathrm{m}>0$ and

$$
0<c\left[\frac{1}{\beta^{\alpha}} \int_{0}^{T}\left(T^{\beta}-\tau^{\beta}\right)^{\alpha-1} \boldsymbol{E}\left(-\frac{a_{p}}{\beta^{\alpha}}\left(T^{\beta}-\tau^{\beta}\right)^{\alpha}\right) \mathcal{Q}(\tau) d\left(\tau^{\beta}\right)\right]^{2}<1
$$

we get

$$
\begin{aligned}
& \sup _{a_{p}>0} \frac{\left[1-\left(1-c\left[\frac{1}{\beta^{\alpha}} \int_{0}^{T}\left(T^{\beta}-\tau^{\beta}\right)^{\alpha-1} \boldsymbol{E}\left(-\frac{a_{p}}{\beta^{\alpha}}\left(T^{\beta}-\tau^{\beta}\right)^{\alpha}\right) \mathcal{Q}(\tau) d\left(\tau^{\beta}\right)\right]^{2}\right)^{\mathrm{m}}\right]^{\theta}}{\frac{1}{\beta^{\alpha}} \int_{0}^{T}\left(T^{\beta}-\tau^{\beta}\right)^{\alpha-1} \boldsymbol{E}\left(-\frac{a_{p}}{\beta^{\alpha}}\left(T^{\beta}-\tau^{\beta}\right)^{\alpha}\right) \mathcal{Q}(\tau) d\left(\tau^{\beta}\right)} \\
& \quad \leq c^{\frac{1}{2}} \mathrm{~m}^{\frac{1}{2}} .
\end{aligned}
$$

Proof We define $\psi(y):=y^{-2}\left[1-\left(1-y^{2}\right)^{\mathrm{m}}\right]^{2 \theta}$, where $y^{2}:=c\left[\frac{1}{\beta^{\alpha}} \int_{0}^{T}\left(T^{\beta}-\tau^{\beta}\right)^{\alpha-1} \boldsymbol{E}\left(-\frac{a_{p}}{\beta^{\alpha}}\left(T^{\beta}-\right.\right.\right.$ $\left.\left.\left.\tau^{\beta}\right)^{\alpha}\right) \mathcal{Q}(\tau) d\left(\tau^{\beta}\right)\right]^{2}$. It is easy to see that the function $\psi(y)$ is continuous in $[0,+\infty)$ when $y \in(0,1)$ and

$$
\begin{aligned}
& \frac{\left[1-\left(1-c\left[\frac{1}{\beta^{\alpha}} \int_{0}^{T}\left(T^{\beta}-\tau^{\beta}\right)^{\alpha-1} \boldsymbol{E}\left(-\frac{a_{p}}{\beta^{\alpha}}\left(T^{\beta}-\tau^{\beta}\right)^{\alpha}\right) \mathcal{Q}(\tau) d\left(\tau^{\beta}\right)\right]^{2}\right)^{\mathrm{m}}\right]^{\theta}}{\frac{1}{\beta^{\alpha}} \int_{0}^{T}\left(T^{\beta}-\tau^{\beta}\right)^{\alpha-1} \boldsymbol{E}\left(-\frac{a_{p}}{\beta^{\alpha}}\left(T^{\beta}-\tau^{\beta}\right)^{\alpha}\right) \mathcal{Q}(\tau) d\left(\tau^{\beta}\right)} \\
& \quad=c^{\frac{1}{2}} \psi^{\frac{1}{2}}(y) .
\end{aligned}
$$

For $\theta \in\left(\frac{1}{2}, 1\right]$ and $y \in(0,1)$, applying Lemma 3.3 of [15], we get $\psi(y) \leq \mathrm{m}$. That infers that inequality (14) is correct.

\subsection{A priori parameter choice rule and convergence estimate}

Let us choose $\mathrm{m}:=\mathrm{m}(\varepsilon)$ such that $\left\|\mathcal{F}_{\mathrm{m}, \theta}^{\varepsilon}(\cdot)-\mathcal{F}(\cdot)\right\|_{\mathcal{L}^{2}(\Omega)} \rightarrow 0$ as $\varepsilon \rightarrow 0$. Using an a priori regularization parameter choice rule, we propose the convergence rate for the fractional Landweber regularized solution $\mathcal{F}_{\mathrm{m}, \theta}^{\varepsilon}$ to $\mathcal{F}$.

Theorem 3.1 Let $\mathcal{Q}:[0, T] \rightarrow \mathbb{R}$ for all $0 \leq t \leq T$ and $\mathscr{H} \in \mathcal{L}^{2}(\Omega)$. Assume that (4) and bound condition (12) hold.

If we choose

$$
\mathrm{m}= \begin{cases}\left\lfloor\left(\frac{\mathcal{P}}{\varepsilon}\right)^{\frac{2}{\kappa+1}}\right\rfloor, & 0<\kappa<2, \\ \left\lfloor\left(\frac{\mathcal{P}}{\varepsilon}\right)^{\frac{2}{3}}\right\rfloor, & \kappa \geq 2\end{cases}
$$

then we get

$$
\left\|\mathcal{F}_{\mathrm{m}, \theta}^{\varepsilon}(\cdot)-\mathcal{F}(\cdot)\right\|_{\mathcal{L}^{2}(\Omega)} \leq \begin{cases}\left(c+\left(c \mathcal{Q}_{0}^{2} \mathcal{M}^{2}\right)^{-\frac{\kappa}{2}}\left(\frac{\kappa}{2}\right)^{\frac{\kappa}{2}}\right) \mathcal{P}^{\frac{1}{\kappa+1}} \varepsilon^{\frac{\kappa}{\kappa+1},}, & 0<\kappa<2, \\ \left(c+\frac{1}{c \mathcal{Q}_{0}^{2} \mathcal{M}^{2}}\right) \mathcal{P}^{\frac{1}{3}} \varepsilon^{\frac{2}{3}}, & \kappa \geq 2,\end{cases}
$$

here $\lfloor\mathrm{m}\rfloor$ represents the largest integer not larger than $\mathrm{m}$. 
Proof From the triangle inequality, we obtain

$$
\left\|\mathcal{F}_{\mathrm{m}, \theta}^{\varepsilon}(\cdot)-\mathcal{F}(\cdot)\right\|_{\mathcal{L}^{2}(\Omega)} \leq\left\|\mathcal{F}_{\mathrm{m}, \theta}^{\varepsilon}(\cdot)-\mathcal{F}_{\mathrm{m}, \theta}(\cdot)\right\|_{\mathcal{L}^{2}(\Omega)}+\left\|\mathcal{F}_{\mathrm{m}, \theta}(\cdot)-\mathcal{F}(\cdot)\right\|_{\mathcal{L}^{2}(\Omega)}
$$

First, we give an estimate for the first term $\left\|\mathcal{F}_{\mathrm{m}, \theta}^{\varepsilon}(\cdot)-\mathcal{F}_{\mathrm{m}, \theta}(\cdot)\right\|_{\mathcal{L}^{2}(\Omega)}$. Applying Lemma 3.1, we get

$$
\begin{aligned}
& \left\|\mathcal{F}_{\mathrm{m}, \theta}^{\varepsilon}(\cdot)-\mathcal{F}_{\mathrm{m}, \theta}(\cdot)\right\|_{\mathcal{L}^{2}(\Omega)} \\
& \leq\left(\sum_{p=1}^{\infty} \frac{\left[1-\left(1-c\left[\frac{1}{\beta^{\alpha}} \int_{0}^{T}\left(T^{\beta}-\tau^{\beta}\right)^{\alpha-1} \boldsymbol{E}\left(-\frac{a_{p}}{\beta^{\alpha}}\left(T^{\beta}-\tau^{\beta}\right)^{\alpha}\right) \mathcal{Q}(\tau) d\left(\tau^{\beta}\right)\right]^{2}\right)^{\mathrm{m}}\right]^{2 \theta}}{\left(\frac{1}{\beta^{\alpha}} \int_{0}^{T}\left(T^{\beta}-\tau^{\beta}\right)^{\alpha-1} \boldsymbol{E}\left(-\frac{a_{p}}{\beta^{\alpha}}\left(T^{\beta}-\tau^{\beta}\right)^{\alpha}\right) \mathcal{Q}(\tau) d\left(\tau^{\beta}\right)\right)^{2}}\right. \\
& \left.\quad \times\left|\Theta_{p}^{\varepsilon}-\Theta_{p}\right|^{2}\right)^{\frac{1}{2}} \\
& \leq \sup _{a_{p}>0} \frac{\left[1-\left(1-c\left[\frac{1}{\beta^{\alpha}} \int_{0}^{T}\left(T^{\beta}-\tau^{\beta}\right)^{\alpha-1} \boldsymbol{E}\left(-\frac{a_{p}}{\beta^{\alpha}}\left(T^{\beta}-\tau^{\beta}\right)^{\alpha}\right) \mathcal{Q}(\tau) d\left(\tau^{\beta}\right)\right]^{2}\right)^{\mathrm{m}}\right]^{\theta}}{\frac{1}{\beta^{\alpha}} \int_{0}^{T}\left(T^{\beta}-\tau^{\beta}\right)^{\alpha-1} \boldsymbol{E}\left(-\frac{a_{p}}{\beta^{\alpha}}\left(T^{\beta}-\tau^{\beta}\right)^{\alpha}\right) \mathcal{Q}(\tau) d\left(\tau^{\beta}\right)} \\
& \quad \times\left\|\Theta^{\varepsilon}-\Theta\right\|_{\mathcal{L}^{2}(\Omega)} \\
& \leq c^{\frac{1}{2}} \mathrm{~m}^{\frac{1}{2}} \varepsilon .
\end{aligned}
$$

Hence,

$$
\left\|\mathcal{F}_{\mathrm{m}, \theta}^{\varepsilon}(\cdot)-\mathcal{F}_{\mathrm{m}, \theta}(\cdot)\right\|_{\mathcal{L}^{2}(\Omega)} \leq c^{\frac{1}{2}} \mathrm{~m}^{\frac{1}{2}} \varepsilon
$$

On the other hand, we estimate the second term

$$
\begin{aligned}
& \left\|\mathcal{F}_{\mathrm{m}, \theta}(\cdot)-\mathcal{F}(\cdot)\right\|_{\mathcal{L}^{2}(\Omega)} \\
& =\| \sum_{p=1}^{\infty} \frac{1-\left[1-\left(1-c\left[\frac{1}{\beta^{\alpha}} \int_{0}^{T}\left(T^{\beta}-\tau^{\beta}\right)^{\alpha-1} \boldsymbol{E}\left(-\frac{a_{p}}{\beta^{\alpha}}\left(T^{\beta}-\tau^{\beta}\right)^{\alpha}\right) \mathcal{Q}(\tau) d\left(\tau^{\beta}\right)\right]^{2}\right)^{\mathrm{m}}\right]^{\theta}}{\frac{1}{\beta^{\alpha}} \int_{0}^{T}\left(T^{\beta}-\tau^{\beta}\right)^{\alpha-1} \boldsymbol{E}\left(-\frac{a_{p}}{\beta^{\alpha}}\left(T^{\beta}-\tau^{\beta}\right)^{\alpha}\right) \mathcal{Q}(\tau) d\left(\tau^{\beta}\right)} \\
& \quad \times\left\langle\Theta, w_{p}\right\rangle w_{p} \|_{\mathcal{L}^{2}(\Omega)} .
\end{aligned}
$$

We note that $\theta \in\left(\frac{1}{2}, 1\right]$, Lemma 2.6 , then

$$
\begin{aligned}
& \left\|\mathcal{F}_{\mathrm{m}, \theta}(\cdot)-\mathcal{F}(\cdot)\right\|_{\mathcal{L}^{2}(\Omega)} \\
& \leq\left\|\sum_{p=1}^{\infty}\left(1-c\left[\frac{1}{a_{p}} \mathcal{Q}_{0} \mathcal{M}\right]^{2}\right)^{\mathrm{m}}\left\langle\mathcal{F}, w_{p}\right\rangle w_{p}\right\|_{\mathcal{L}^{2}(\Omega)} \\
& \quad \leq\left(\sum_{p=1}^{\infty}\left(1-c\left[\frac{1}{a_{p}} \mathcal{Q}_{0} \mathcal{M}\right]^{2}\right)^{2 \mathrm{~m}} a_{p}^{-2 \kappa} a_{p}^{2 \kappa}\left|\left\langle\mathcal{F}, w_{p}\right\rangle\right|^{2}\right)^{\frac{1}{2}} .
\end{aligned}
$$

Apply Lemma 2.4

$$
\left\|\mathcal{F}_{\mathrm{m}, \theta}(\cdot)-\mathcal{F}(\cdot)\right\|_{\mathcal{L}^{2}(\Omega)} \leq\left(c \mathcal{Q}_{0}^{2} \mathcal{M}^{2}\right)^{-\frac{\kappa}{2}}\left(\frac{\kappa}{2}\right)^{\frac{\kappa}{2}} \mathrm{~m}^{-\frac{\kappa}{2}} \mathcal{P}
$$


Thus

$$
\left\|\mathcal{F}_{\mathrm{m}, \theta}^{\varepsilon}(\cdot)-\mathcal{F}(\cdot)\right\|_{\mathcal{L}^{2}(\Omega)} \leq c^{\frac{1}{2}} \mathrm{~m}^{\frac{1}{2}} \varepsilon+\left(c \mathcal{Q}_{0}^{2} \mathcal{M}^{2}\right)^{-\frac{\kappa}{2}}\left(\frac{\kappa}{2}\right)^{\frac{\kappa}{2}} \mathrm{~m}^{-\frac{\kappa}{2}} \mathcal{P} .
$$

Choose the regularization parameter $\mathrm{m}$ by

$$
\mathrm{m}= \begin{cases}\left\lfloor\left(\frac{\mathcal{P}}{\varepsilon}\right)^{\frac{2}{\kappa+1}}\right\rfloor, & 0<\kappa<2, \\ \left\lfloor\left(\frac{\mathcal{P}}{\varepsilon}\right)^{\frac{2}{3}}\right\rfloor, & \kappa \geq 2,\end{cases}
$$

then we have

$$
\left\|\mathcal{F}_{\mathrm{m}, \theta}^{\varepsilon}(\cdot)-\mathcal{F}(\cdot)\right\|_{\mathcal{L}^{2}(\Omega)} \leq \begin{cases}\left(\sqrt{c}+\left(c \mathcal{Q}_{0}^{2} \mathcal{M}^{2}\right)^{-\frac{\kappa}{2}}\left(\frac{\kappa}{2}\right)^{\frac{\kappa}{2}}\right) \mathcal{P}^{\frac{1}{\kappa+1}} \varepsilon^{\frac{\kappa}{\kappa+1}}, & 0<\kappa<2, \\ \left(\sqrt{c}+\frac{1}{c \mathcal{Q}_{0}^{2} \mathcal{M}^{2}}\right) \mathcal{P}^{\frac{1}{3}} \varepsilon^{\frac{2}{3}}, & \kappa \geq 2 .\end{cases}
$$

This ends the proof.

\subsection{A posteriori parameter choice rule and convergence estimate}

Now, we consider an a posteriori regularization choice rule called Morozov's discrepancy principle [9], we choose the regularization parameter $m$ such that

$$
\left\|\mathcal{K} \mathcal{F}_{\mathrm{m}, \theta}^{\varepsilon}-\Theta^{\varepsilon}\right\|_{\mathcal{L}^{2}(\Omega)} \leq \vartheta \varepsilon \leq\left\|\mathcal{K} \mathcal{F}_{\mathrm{m}-1, \theta}^{\varepsilon}-\Theta^{\varepsilon}\right\|_{\mathcal{L}^{2}(\Omega)}
$$

where $\left\|\Theta^{\varepsilon}\right\|_{\mathcal{L}^{2}(\Omega)} \geq \vartheta \varepsilon$, $\vartheta$, which makes (17) hold at the first iterator time, is a constant independent of $\varepsilon$.

Choose $\vartheta>1$, and the bound for $m$ is given and depends on $\varepsilon$ and $\mathcal{P}$.

Lemma 3.2 If m satisfies (17), we can get the following inequality:

$$
\mathrm{m} \leq \begin{cases}\frac{\kappa+1}{2 c \mathcal{Q}_{0}^{2} \mathcal{M}^{2}}\left(\frac{\overline{\mathbb{M}_{\alpha, \beta}^{+}}\|\mathcal{Q}\|_{\infty}}{\vartheta-1}\right)^{\frac{2}{\kappa+1}}\left(\frac{\mathcal{P}}{\varepsilon}\right)^{\frac{2}{\kappa+1}}, & 0<\kappa<1 \\ \frac{\kappa+1}{2 c \mathcal{Q}_{0}^{2} \mathcal{M}^{2}} \frac{\overline{\mathbb{M}_{\alpha, \beta}^{+}}\|\mathcal{Q}\|_{\infty}}{\vartheta-1} \frac{\mathcal{P}}{\varepsilon}, & \kappa \geq 1 .\end{cases}
$$

Proof From (17), we have

$$
\begin{aligned}
\vartheta \varepsilon & =\left\|\mathcal{K} \mathcal{F}_{\mathrm{m}-1, \theta}^{\varepsilon}-\Theta^{\varepsilon}\right\|_{\mathcal{L}^{2}(\Omega)} \\
\leq & \| \sum_{p=1}^{\infty}\left(\left[1-\left(1-c\left[\frac{1}{\beta^{\alpha}} \int_{0}^{T}\left(T^{\beta}-\tau^{\beta}\right)^{\alpha-1} \boldsymbol{E}\left(-\frac{a_{p}}{\beta^{\alpha}}\left(T^{\beta}-\tau^{\beta}\right)^{\alpha}\right) \mathcal{Q}(\tau) d\left(\tau^{\beta}\right)\right]^{2}\right)^{\mathrm{m}-1}\right]^{\theta}\right. \\
& -1) \Theta_{p}^{\varepsilon} w_{p} \|_{\mathcal{L}^{2}(\Omega)}
\end{aligned}
$$

By $\theta \in\left(\frac{1}{2}, 1\right]$ and $0<c\left[\frac{1}{\beta^{\alpha}} \int_{0}^{T}\left(T^{\beta}-\tau^{\beta}\right)^{\alpha-1} \boldsymbol{E}\left(-\frac{a_{p}}{\beta^{\alpha}}\left(T^{\beta}-\tau^{\beta}\right)^{\alpha}\right) \mathcal{Q}(\tau) d\left(\tau^{\beta}\right)\right]^{2}<1$, we get

$$
\vartheta \varepsilon=\left\|\mathcal{K} \mathcal{F}_{\mathrm{m}-1, \theta}^{\varepsilon}-\Theta^{\varepsilon}\right\|_{\mathcal{L}^{2}(\Omega)} \leq\left\|\Theta^{\varepsilon}-\Theta\right\|_{\mathcal{L}^{2}(\Omega)}+\mathcal{I}
$$


where

$$
\begin{aligned}
\mathcal{I}= & \| \sum_{p=1}^{\infty}\left(1-c\left[\frac{1}{\beta^{\alpha}} \int_{0}^{T}\left(T^{\beta}-\tau^{\beta}\right)^{\alpha-1} \boldsymbol{E}\left(-\frac{a_{p}}{\beta^{\alpha}}\left(T^{\beta}-\tau^{\beta}\right)^{\alpha}\right) \mathcal{Q}(\tau) d\left(\tau^{\beta}\right)\right]^{2}\right)^{\mathrm{m}-1} \\
& \times\left\langle\Theta, w_{p}\right\rangle w_{p} \|_{\mathcal{L}^{2}(\Omega)} .
\end{aligned}
$$

In view of Lemma 2.6 and (11), we obtain

$$
\begin{aligned}
& \mathcal{I} \leq\left\|\sum_{p=1}^{\infty}\left(1-c\left[\frac{1}{a_{p}} \mathcal{Q}_{0} \mathcal{M}\right]^{2}\right)^{\mathrm{m}-1}\left\langle\Theta, w_{p}\right\rangle w_{p}\right\|_{\mathcal{L}^{2}(\Omega)} \\
& \leq\left(\sum_{p=1}^{\infty}\left(1-c\left[\frac{1}{a_{p}} \mathcal{Q}_{0} \mathcal{M}\right]^{2}\right)^{2 \mathrm{~m}-2} a_{p}^{-2 \kappa} a_{p}^{2 \kappa}\left|\left\langle\Theta, w_{p}\right\rangle\right|^{2}\right)^{\frac{1}{2}} \\
& \leq\left(\sum_{p=1}^{\infty}\left(1-c\left[\frac{1}{a_{p}} \mathcal{Q}_{0} \mathcal{M}\right]^{2}\right)^{2 \mathrm{~m}-2} \frac{{\overline{\mathbb{M}_{\alpha, \beta}^{+}}}^{2}\|\mathcal{Q}\|_{\infty}^{2}}{a_{p}^{2 \kappa+2}} a_{p}^{2 \kappa}\left|\left\langle\mathcal{F}, w_{p}\right\rangle\right|^{2}\right)^{\frac{1}{2}} \text {. }
\end{aligned}
$$

By Lemma 2.4, this implies that

$$
\begin{aligned}
\mathcal{I} & \leq \sup _{a_{p}>0}\left(1-c\left[\frac{1}{a_{p}} \mathcal{Q}_{0} \mathcal{M}\right]^{2}\right)^{\mathrm{m}-1} \frac{\overline{\mathbb{M}_{\alpha, \beta}^{+}}\|\mathcal{Q}\|_{\infty}}{a_{p}^{\kappa+1}}\|\mathcal{F}\|_{\mathcal{D}\left((-\mathcal{B})^{-\kappa}\right)} \\
& \leq \frac{\overline{\mathbb{M}_{\alpha, \beta}^{+}}\|\mathcal{Q}\|_{\infty}}{\left(c \mathcal{Q}_{0}^{2} \mathcal{M}^{2}\right)^{\frac{\kappa+1}{2}}}\left(\frac{\kappa+1}{2}\right)^{\frac{\kappa+1}{2}} \mathrm{~m}^{-\frac{\kappa+1}{2}} \mathcal{P} .
\end{aligned}
$$

From the above results we have

$$
(\vartheta-1) \varepsilon \leq \overline{\mathbb{M}_{\alpha, \beta}^{+}}\|\mathcal{Q}\|_{\infty}\left(\frac{\kappa+1}{2 c \mathcal{Q}_{0}^{2} \mathcal{M}^{2}}\right)^{\frac{\kappa+1}{2}} \mathrm{~m}^{-\frac{\kappa+1}{2}} \mathcal{P}
$$

This yields

$$
\mathrm{m} \leq \begin{cases}\frac{\kappa+1}{2 c \mathcal{Q}_{0}^{2} \mathcal{M}^{2}}\left(\frac{\overline{\mathbb{M}_{\alpha, \beta}^{+}}\|\mathcal{Q}\|_{\infty}}{\vartheta-1}\right)^{\frac{2}{\kappa+1}}\left(\frac{\mathcal{P}}{\varepsilon}\right) \frac{2}{\kappa+1}, & 0<\kappa<1, \\ \frac{\kappa+1}{2 c \mathcal{Q}_{0}^{2} \mathcal{M}^{2}} \frac{\overline{\mathbb{M}_{\alpha, \beta}^{+}}\|\mathcal{Q}\|_{\infty}}{\vartheta-1} \frac{\mathcal{P}}{\varepsilon}, & \kappa \geq 1 .\end{cases}
$$

Theorem 3.2 We recall that $\mathrm{m}$ in Lemma 3.2 and bound condition (12) hold. Then we have

$$
\begin{aligned}
& \left\|\mathcal{F}_{\mathrm{m}, \boldsymbol{\theta}}^{\varepsilon}(\cdot)-\mathcal{F}(\cdot)\right\|_{\mathcal{L}^{2}(\Omega)} \\
& \leq\left(\frac{1+\vartheta}{\mathcal{Q}_{0} \mathcal{M}}\right)^{\frac{\kappa}{\kappa+1}} \mathcal{P}^{\frac{1}{\kappa+1}} \mathcal{E}^{\frac{\kappa}{\kappa+1}}+ \begin{cases}c^{\frac{1}{2}}\left(\frac{\kappa+1}{2 c \mathcal{Q}_{0}^{2} \mathcal{M}^{2}}\right)^{\frac{1}{2}}\left(\frac{\overline{\mathbb{M}_{\alpha, \beta}^{+}}\|\mathcal{Q}\|_{\infty}}{\vartheta-1}\right)^{\frac{1}{\kappa+1}} \mathcal{P} \frac{1}{\kappa+1} \frac{\kappa}{\mathcal{C}^{\kappa+1}}, & 0<\kappa<1, \\
\left(c \frac{\kappa+1}{2 c \mathcal{Q}_{0}^{2} \mathcal{M}^{2}} \frac{\overline{\mathbb{M}}_{\alpha, \beta}^{+}\|\mathcal{Q}\|_{\infty}}{\vartheta-1} \mathcal{P} \varepsilon\right)^{\frac{1}{2}}, & \kappa \geq 1 .\end{cases}
\end{aligned}
$$

Proof From the triangle inequality, we obtain

$$
\left\|\mathcal{F}_{\mathrm{m}, \theta}^{\varepsilon}(\cdot)-\mathcal{F}(\cdot)\right\|_{\mathcal{L}^{2}(\Omega)} \leq\left\|\mathcal{F}_{\mathrm{m}, \theta}^{\varepsilon}(\cdot)-\mathcal{F}_{\mathrm{m}, \theta}(\cdot)\right\|_{\mathcal{L}^{2}(\Omega)}+\left\|\mathcal{F}_{\mathrm{m}, \theta}(\cdot)-\mathcal{F}(\cdot)\right\|_{\mathcal{L}^{2}(\Omega)} .
$$


In view of (16) and Lemma 3.2, we deduce that

$$
\begin{aligned}
& \left\|\mathcal{F}_{\mathrm{m}, \theta}^{\varepsilon}(\cdot)-\mathcal{F}_{\mathrm{m}, \theta}(\cdot)\right\|_{\mathcal{L}^{2}(\Omega)} \leq c^{\frac{1}{2} \mathrm{~m}^{\frac{1}{2}} \varepsilon}
\end{aligned}
$$

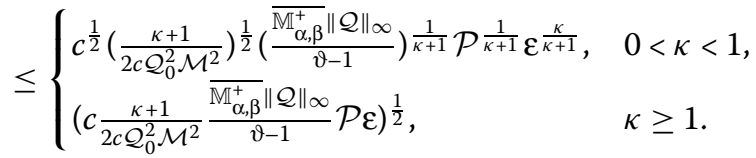

Now we give the bound for the second term. Same as above, we have

$$
\begin{aligned}
& \left\|\mathcal{F}_{\mathrm{m}, \theta}(\cdot)-\mathcal{F}(\cdot)\right\|_{\mathcal{L}^{2}(\Omega)} \\
& =\| \sum_{p=1}^{\infty} \frac{1-\left[1-\left(1-c\left[\frac{1}{\beta^{\alpha}} \int_{0}^{T}\left(T^{\beta}-\tau^{\beta}\right)^{\alpha-1} \boldsymbol{E}\left(-\frac{a_{p}}{\beta^{\alpha}}\left(T^{\beta}-\tau^{\beta}\right)^{\alpha}\right) \mathcal{Q}(\tau) d\left(\tau^{\beta}\right)\right]^{2}\right)^{\mathrm{m}}\right]^{\theta}}{\frac{1}{\beta^{\alpha}} \int_{0}^{T}\left(T^{\beta}-\tau^{\beta}\right)^{\alpha-1} \boldsymbol{E}\left(-\frac{a_{p}}{\beta^{\alpha}}\left(T^{\beta}-\tau^{\beta}\right)^{\alpha}\right) \mathcal{Q}(\tau) d\left(\tau^{\beta}\right)} \\
& \quad \times \Theta_{p} w_{p} \|_{\mathcal{L}^{2}(\Omega)} \cdot
\end{aligned}
$$

Applying Hölder's inequality, we have

$$
\begin{aligned}
& \left\|\mathcal{F}_{\mathrm{m}, \theta}(\cdot)-\mathcal{F}(\cdot)\right\|_{\mathcal{L}^{2}(\Omega)} \\
& =\mathcal{J} \| \sum_{p=1}^{\infty} \frac{1-\left[1-\left(1-c\left[\frac{1}{\beta^{\alpha}} \int_{0}^{T}\left(T^{\beta}-\tau^{\beta}\right)^{\alpha-1} \boldsymbol{E}\left(-\frac{a_{p}}{\beta^{\alpha}}\left(T^{\beta}-\tau^{\beta}\right)^{\alpha}\right) \mathcal{Q}(\tau) d\left(\tau^{\beta}\right)\right]^{2}\right)^{\mathrm{m}}\right]^{\theta}}{\frac{1}{\beta^{\alpha}} \int_{0}^{T}\left(T^{\beta}-\tau^{\beta}\right)^{\alpha-1} \boldsymbol{E}\left(-\frac{a_{p}}{\beta^{\alpha}}\left(T^{\beta}-\tau^{\beta}\right)^{\alpha}\right) \mathcal{Q}(\tau) d\left(\tau^{\beta}\right)} \\
& \quad \times \Theta_{p} w_{p} \|_{\mathcal{L}^{2}(\Omega)}^{\frac{\kappa}{\kappa+1}},
\end{aligned}
$$

where

$$
\begin{aligned}
\mathcal{J}= & \| \sum_{p=1}^{\infty} 1-\left[1-\left(1-c\left[\frac{1}{\beta^{\alpha}} \int_{0}^{T}\left(T^{\beta}-\tau^{\beta}\right)^{\alpha-1} \boldsymbol{E}\left(-\frac{a_{p}}{\beta^{\alpha}}\left(T^{\beta}-\tau^{\beta}\right)^{\alpha}\right) \mathcal{Q}(\tau) d\left(\tau^{\beta}\right)\right]^{2}\right)^{\mathrm{m}}\right]^{\theta} \\
& \times \mathcal{F}_{p} w_{p} \|_{\mathcal{L}^{2}(\Omega)}^{\frac{1}{\kappa+1}} .
\end{aligned}
$$

From $\theta \in\left(\frac{1}{2}, 1\right]$ and $0<c\left[\frac{1}{\beta^{\alpha}} \int_{0}^{T}\left(T^{\beta}-\tau^{\beta}\right)^{\alpha-1} \boldsymbol{E}\left(-\frac{a_{p}}{\beta^{\alpha}}\left(T^{\beta}-\tau^{\beta}\right)^{\alpha}\right) \mathcal{Q}(\tau) d\left(\tau^{\beta}\right)\right]^{2}<1$, we deduce that

$$
\mathcal{J} \leq\left\|\sum_{p=1}^{\infty} \mathcal{F}_{p} w_{p}\right\|_{\mathcal{L}^{2}(\Omega)}^{\frac{1}{\kappa+1}} \leq \sup _{a_{p}>0}\left(\frac{1}{a_{p}}\right)^{\frac{\kappa}{\kappa+1}}\|\mathcal{F}\|_{\mathcal{D}\left((-\mathscr{B})^{-\kappa}\right)}^{\frac{1}{\kappa+1}} .
$$

Hence, we obtain

$$
\begin{aligned}
& \left\|\mathcal{F}_{\mathrm{m}, \theta}(\cdot)-\mathcal{F}(\cdot)\right\|_{\mathcal{L}^{2}(\Omega)} \\
& \quad \leq \sup _{a_{p}>0}\left(\frac{1}{a_{p} \frac{1}{\beta^{\alpha}} \int_{0}^{T}\left(T^{\beta}-\tau^{\beta}\right)^{\alpha-1} \boldsymbol{E}\left(-\frac{a_{p}}{\beta^{\alpha}}\left(T^{\beta}-\tau^{\beta}\right)^{\alpha}\right) \mathcal{Q}(\tau) d\left(\tau^{\beta}\right)}\right)^{\frac{\kappa}{\kappa+1}}
\end{aligned}
$$




$$
\begin{aligned}
& \times \mathcal{P}^{\frac{1}{\kappa+1}}\left(\left\|\sum_{p=1}^{\infty}\left\langle\Theta-\Theta^{\varepsilon}, w_{p}\right) w_{p}\right\|_{\mathcal{L}^{2}(\Omega)}\right. \\
& +\| \sum_{p=1}^{\infty}(1-[1-(1 \\
& \left.\left.\left.\left.-c\left[\frac{1}{\beta^{\alpha}} \int_{0}^{T}\left(T^{\beta}-\tau^{\beta}\right)^{\alpha-1} \boldsymbol{E}\left(-\frac{a_{p}}{\beta^{\alpha}}\left(T^{\beta}-\tau^{\beta}\right)^{\alpha}\right) \mathcal{Q}(\tau) d\left(\tau^{\beta}\right)\right]^{2}\right)^{\mathrm{m}}\right]^{\theta}\right) \Theta_{p}^{\varepsilon} w_{p} \|\right)^{\frac{\kappa}{\kappa+1}} .
\end{aligned}
$$

In view of (17), we have

$$
\left\|\mathcal{F}_{\mathrm{m}, \theta}(\cdot)-\mathcal{F}(\cdot)\right\|_{\mathcal{L}^{2}(\Omega)} \leq\left(\frac{1+\vartheta}{\mathcal{Q}_{0} \mathcal{M}}\right)^{\frac{\kappa}{\kappa+1}} \mathcal{P}^{\frac{1}{\kappa+1}} \mathcal{E}^{\frac{\kappa}{\kappa+1}} .
$$

Hence, we get

$$
\begin{aligned}
& \left\|\mathcal{F}_{\mathrm{m}, \theta}^{\varepsilon}(\cdot)-\mathcal{F}(\cdot)\right\|_{\mathcal{L}^{2}(\Omega)} \\
& \quad \leq\left(\frac{1+\vartheta}{\mathcal{Q}_{0} \mathcal{M}}\right)^{\frac{\kappa}{\kappa+1}} \mathcal{P}^{\frac{1}{\kappa+1}} \mathcal{E}^{\frac{\kappa}{\kappa+1}}+ \begin{cases}c^{\frac{1}{2}}\left(\frac{\kappa+1}{2 c \mathcal{Q}_{0}^{2} \mathcal{M}^{2}}\right)^{\frac{1}{2}}\left(\frac{\overline{\mathbb{M}_{\alpha, \beta}^{+}}\|\mathcal{Q}\|_{\infty}}{\vartheta-1}\right)^{\frac{1}{\kappa+1}} \mathcal{P}^{\frac{1}{\kappa+1}} \mathcal{E}^{\frac{\kappa}{\kappa+1}}, & 0<\kappa<1, \\
\left(c \frac{\kappa+1}{2 c \mathcal{Q}_{0}^{2} \mathcal{M}^{2}} \frac{\overline{\mathbb{M}}_{\alpha, \beta}^{+} \| \mathcal{Q}_{\infty}}{\vartheta-1} \mathcal{P} \varepsilon\right)^{\frac{1}{2}}, & \kappa \geq 1 .\end{cases}
\end{aligned}
$$

This ends the proof.

\section{Fractional Tikhonov regularization method and convergence rate}

Now, we propose another method to solve the ill-posed problem (1) satisfying (3), that is, a fractional Tikhonov regularization method. Besides, the convergence analysis between the exact solution $\mathcal{F}$ and the fractional Tikhonov regularized solution $\mathcal{F}_{n, \theta}^{\varepsilon}$ are also considered.

From [15], the fractional Tikhonov regularization solution is given by

$$
\mathcal{F}_{\mathrm{n}, \theta}(\mathrm{x})=\sum_{p=1}^{\infty} \frac{\left(\frac{1}{\beta^{\alpha}} \int_{0}^{T}\left(T^{\beta}-\tau^{\beta}\right)^{\alpha-1} \boldsymbol{E}\left(-\frac{a_{p}}{\beta^{\alpha}}\left(T^{\beta}-\tau^{\beta}\right)^{\alpha}\right) \mathcal{Q}(\tau) d\left(\tau^{\beta}\right)\right)^{2 \theta-1}}{\left[\left(\frac{1}{\beta^{\alpha}} \int_{0}^{T}\left(T^{\beta}-\tau^{\beta}\right)^{\alpha-1} \boldsymbol{E}\left(-\frac{a_{p}}{\beta^{\alpha}}\left(T^{\beta}-\tau^{\beta}\right)^{\alpha}\right) \mathcal{Q}(\tau) d\left(\tau^{\beta}\right)\right)^{2}+\mathrm{n}\right]^{\theta}}\left\langle\Theta, w_{p}\right\rangle w_{p} .
$$

If the observation data $\mathscr{H}$ are noised by $\mathscr{H}^{\varepsilon}$, then we have

$$
\begin{aligned}
& \mathcal{F}_{\mathrm{n}, \theta}^{\varepsilon}(\mathrm{x}) \\
& \quad=\sum_{p=1}^{\infty} \frac{\left(\frac{1}{\beta^{\alpha}} \int_{0}^{T}\left(T^{\beta}-\tau^{\beta}\right)^{\alpha-1} \boldsymbol{E}\left(-\frac{a_{p}}{\beta^{\alpha}}\left(T^{\beta}-\tau^{\beta}\right)^{\alpha}\right) \mathcal{Q}(\tau) d\left(\tau^{\beta}\right)\right)^{2 \theta-1}}{\left[\left(\frac{1}{\beta^{\alpha}} \int_{0}^{T}\left(T^{\beta}-\tau^{\beta}\right)^{\alpha-1} \boldsymbol{E}\left(-\frac{a_{p}}{\beta^{\alpha}}\left(T^{\beta}-\tau^{\beta}\right)^{\alpha}\right) \mathcal{Q}(\tau) d\left(\tau^{\beta}\right)\right)^{2}+\mathrm{n}\right]^{\theta}}\left\langle\Theta^{\varepsilon}, w_{p}\right\rangle w_{p},
\end{aligned}
$$

where the regularization parameter $\mathrm{n}>0$, and $\theta \in\left(\frac{1}{2}, 1\right]$ is the fractional order. We note that when $\theta=1$, the fractional Tikhonov method becomes a standard Tikhonov regularization.

Lemma 4.1 Let $\theta \in\left(\frac{1}{2}, 1\right], n>0$, we get

$$
\sup _{a_{p}>0} \frac{\left(\frac{1}{\beta^{\alpha}} \int_{0}^{T}\left(T^{\beta}-\tau^{\beta}\right)^{\alpha-1} \boldsymbol{E}\left(-\frac{a_{p}}{\beta^{\alpha}}\left(T^{\beta}-\tau^{\beta}\right)^{\alpha}\right) \mathcal{Q}(\tau) d\left(\tau^{\beta}\right)\right)^{2 \theta-1}}{\left[\left(\frac{1}{\beta^{\alpha}} \int_{0}^{T}\left(T^{\beta}-\tau^{\beta}\right)^{\alpha-1} \boldsymbol{E}\left(-\frac{a_{p}}{\beta^{\alpha}}\left(T^{\beta}-\tau^{\beta}\right)^{\alpha}\right) \mathcal{Q}(\tau) d\left(\tau^{\beta}\right)\right)^{2}+n\right]^{\theta}} \leq C_{\theta}^{\frac{1}{2}} n^{-\frac{1}{2}} .
$$


Proof We define $\psi(y):=y^{-2}\left(\frac{y^{2}}{y^{2}+n}\right)^{2 \chi}$, where

$$
y:=\frac{1}{\beta^{\alpha}} \int_{0}^{T}\left(T^{\beta}-\tau^{\beta}\right)^{\alpha-1} \boldsymbol{E}\left(-\frac{a_{p}}{\beta^{\alpha}}\left(T^{\beta}-\tau^{\beta}\right)^{\alpha}\right) \mathcal{Q}(\tau) d\left(\tau^{\beta}\right) .
$$

By proving similarly Lemma 3.1 in [15], we get

$$
\psi(y) \leq \psi\left(y^{*}\right)=\mathrm{n}^{-1} C_{\chi}
$$

\subsection{A priori parameter choice rule and convergence estimate}

Let us choose a regularization parameter $\mathrm{n}$, which depends on $\varepsilon$ so that if $\varepsilon \rightarrow 0$, then we get $\left\|\mathcal{F}_{\mathrm{n}, \theta}^{\varepsilon}(\cdot)-\mathcal{F}(\cdot)\right\|_{\mathcal{L}^{2}(\Omega)}$ tends to 0 . The convergence rate for the regularized solution $\mathcal{F}_{\mathrm{n}, \theta}^{\varepsilon}$ to the exact solution $\mathcal{F}$ can be got under an a priori regularization parameter choice rule.

Theorem 4.1 Let $\mathscr{H} \in \mathcal{L}^{2}(\Omega)$ and $\mathcal{Q}:[0, T] \rightarrow \mathbb{R}$ for all $0 \leq t \leq T$. Assume that assumption (4) and a priori bound condition (12) hold.

If we choose

$$
n= \begin{cases}\left\lfloor\left(\frac{\varepsilon}{\mathcal{P}}\right)^{\frac{2}{\kappa+1}}\right\rfloor, & 0<\kappa<2, \\ \left\lfloor\left(\frac{\varepsilon}{\mathcal{P}}\right)^{\frac{2}{3}}\right\rfloor, & \kappa \geq 2\end{cases}
$$

which is the regularization parameter, then we get

$$
\left\|\mathscr{F}_{n, \theta}^{\varepsilon}(\cdot)-\mathcal{F}(\cdot)\right\|_{\mathcal{L}^{2}(\Omega)} \leq \begin{cases}\left(C_{\theta}^{\frac{1}{2}}+\mathcal{N}_{1}\left(\kappa, \mathcal{M}^{2}\right)\right) \mathcal{P}^{\frac{1}{\kappa+1}} \varepsilon^{\frac{\kappa}{\kappa+1}}, & 0<\kappa<2, \\ \left(C_{\theta}^{\frac{1}{2}}+\mathcal{N}_{2}\left(\kappa, \mathcal{M}^{2}\right)\right) \mathcal{P}^{\frac{1}{3}} \varepsilon^{\frac{2}{3}}, & \kappa \geq 2,\end{cases}
$$

here $\lfloor n\rfloor$ represents the largest integer not larger than $n$.

Proof From the triangle inequality, we obtain

$$
\left\|\mathcal{F}_{\mathrm{n}, \theta}^{\varepsilon}(\cdot)-\mathcal{F}(\cdot)\right\|_{\mathcal{L}^{2}(\Omega)} \leq\left\|\mathcal{F}_{\mathrm{n}, \theta}^{\varepsilon}(\cdot)-\mathcal{F}_{\mathrm{n}, \theta}(\cdot)\right\|_{\mathcal{L}^{2}(\Omega)}+\left\|\mathcal{F}_{\mathrm{n}, \theta}(\cdot)-\mathcal{F}(\cdot)\right\|_{\mathcal{L}^{2}(\Omega)} .
$$

First, we obtain

$$
\begin{aligned}
& \left\|\mathcal{F}_{\mathrm{n}, \theta}^{\varepsilon}(\cdot)-\mathcal{F}_{\mathrm{n}, \theta}(\cdot)\right\|_{\mathcal{L}^{2}(\Omega)} \\
& \quad \leq\left(\sum_{p=1}^{\infty} \frac{\left(\frac{1}{\beta^{\alpha}} \int_{0}^{T}\left(T^{\beta}-\tau^{\beta}\right)^{\alpha-1} \boldsymbol{E}\left(-\frac{a_{p}}{\beta^{\alpha}}\left(T^{\beta}-\tau^{\beta}\right)^{\alpha}\right) \mathcal{Q}(\tau) d\left(\tau^{\beta}\right)\right)^{2(2 \theta-1)}}{\left[\left(\frac{1}{\beta^{\alpha}} \int_{0}^{T}\left(T^{\beta}-\tau^{\beta}\right)^{\alpha-1} \boldsymbol{E}\left(-\frac{a_{p}}{\beta^{\alpha}}\left(T^{\beta}-\tau^{\beta}\right)^{\alpha}\right) \mathcal{Q}(\tau) d\left(\tau^{\beta}\right)\right)^{2}+\mathrm{n}\right]^{2 \theta}} \mid\left\langle\Theta^{\varepsilon}-\Theta, w_{p} \|^{2}\right)^{\frac{1}{2}}\right. \\
& \quad \leq \sup _{a_{p}>0} \frac{\left(\frac{1}{\beta^{\alpha}} \int_{0}^{T}\left(T^{\beta}-\tau^{\beta}\right)^{\alpha-1} \boldsymbol{E}\left(-\frac{a_{p}}{\beta^{\alpha}}\left(T^{\beta}-\tau^{\beta}\right)^{\alpha}\right) \mathcal{Q}(\tau) d\left(\tau^{\beta}\right)\right)^{2 \theta-1}}{\left.\left.\beta^{\alpha} \int_{0}^{T}\left(T^{\beta}-\tau^{\beta}\right)^{\alpha-1} \boldsymbol{E}\left(-\frac{a_{p}}{\beta^{\alpha}}\left(T^{\beta}-\tau^{\beta}\right)^{\alpha}\right) \mathcal{Q}(\tau) d\left(\tau^{\beta}\right)\right)^{2}+\mathrm{n}\right]^{\theta}}\left\|\Theta^{\varepsilon}-\Theta\right\|_{\mathcal{L}^{2}(\Omega)} \\
& \quad \leq C_{\theta}^{\frac{1}{2}} \mathrm{n}^{-\frac{1}{2}} \varepsilon .
\end{aligned}
$$


Moreover, from (11) we obtain

$$
\begin{aligned}
& \left\|\mathcal{F}_{\mathrm{n}, \theta}(\cdot)-\mathcal{F}(\cdot)\right\|_{\mathcal{L}^{2}(\Omega)} \\
& =\left(\sum_{p=1}^{\infty}\left(1-\frac{\left(\frac{1}{\beta^{\alpha}} \int_{0}^{T}\left(T^{\beta}-\tau^{\beta}\right)^{\alpha-1} \boldsymbol{E}\left(-\frac{a_{p}}{\beta^{\alpha}}\left(T^{\beta}-\tau^{\beta}\right)^{\alpha}\right) \mathcal{Q}(\tau) d\left(\tau^{\beta}\right)\right)^{2 \theta}}{\left[\left(\frac{1}{\beta^{\alpha}} \int_{0}^{T}\left(T^{\beta}-\tau^{\beta}\right)^{\alpha-1} \boldsymbol{E}\left(-\frac{a_{p}}{\beta^{\alpha}}\left(T^{\beta}-\tau^{\beta}\right)^{\alpha}\right) \mathcal{Q}(\tau) d\left(\tau^{\beta}\right)\right)^{2}+\mathrm{n}\right]^{\theta}}\right)^{2}\right. \\
& \left.\quad \times\left|\left\langle\mathcal{F}, w_{p}\right\rangle\right|^{2}\right)^{\frac{1}{2}} .
\end{aligned}
$$

Using the inequality $c_{1}^{5}-c_{2}^{\varsigma} \leq\left(c_{1}-c_{2}\right)^{\varsigma}$, for $0 \leq c_{2} \leq c_{1}, 0 \leq \varsigma \leq 1$, we deduce that

$$
\begin{aligned}
& \left\|\mathcal{F}_{\mathrm{n}, \theta}(\cdot)-\mathcal{F}(\cdot)\right\|_{\mathcal{L}^{2}(\Omega)} \\
& \quad \leq\left(\sum_{p=1}^{\infty}\left(\frac{\mathrm{n}^{\theta}}{\left[\left(\frac{1}{\beta^{\alpha}} \int_{0}^{T}\left(T^{\beta}-\tau^{\beta}\right)^{\alpha-1} \boldsymbol{E}\left(-\frac{a_{p}}{\beta^{\alpha}}\left(T^{\beta}-\tau^{\beta}\right)^{\alpha}\right) \mathcal{Q}(\tau) d\left(\tau^{\beta}\right)\right)^{2}+\mathrm{n}\right]^{\theta}}\right)^{2}\left|\left\langle\mathcal{F}, w_{p}\right\rangle\right|^{2}\right)^{\frac{1}{2}} .
\end{aligned}
$$

Applying Lemma 2.6, we get

$$
\begin{aligned}
& \left\|\mathcal{F}_{\mathrm{n}, \theta}(\cdot)-\mathcal{F}(\cdot)\right\|_{\mathcal{L}^{2}(\Omega)} \\
& \leq\left(\sum_{p=1}^{\infty}\left(\frac{a_{p}^{-\kappa} \mathrm{n}^{\theta}}{\left[\left(\frac{1}{\beta^{\alpha}} \int_{0}^{T}\left(T^{\beta}-\tau^{\beta}\right)^{\alpha-1} \boldsymbol{E}\left(-\frac{a_{p}}{\beta^{\alpha}}\left(T^{\beta}-\tau^{\beta}\right)^{\alpha}\right) \mathcal{Q}(\tau) d\left(\tau^{\beta}\right)\right)^{2}+\mathrm{n}\right]^{\theta}}\right)^{2} a_{p}^{2 \kappa}\right. \\
& \left.\quad \times\left|\left\langle\mathcal{F}, w_{p}\right\rangle\right|^{2}\right)^{\frac{1}{2}} \\
& \leq \sup _{a_{p}>0}\left(a_{p}^{2-\kappa}\left[\frac{\mathrm{n}}{\mathcal{Q}_{0}^{2} \mathcal{M}^{2}+a_{p}^{2} \mathrm{n}}\right]^{\theta}\right)\|\mathcal{F}\|_{\mathcal{D}\left((-\mathcal{B})^{-\kappa}\right) .}
\end{aligned}
$$

From a priori bound on the final data $\|\mathcal{F}\|_{\mathcal{D}\left((-\mathcal{B})^{-\kappa}\right)} \leq \mathcal{P}$ for any $\kappa>0, \theta \in\left(\frac{1}{2}, 1\right]$ and Lemma 2.5, we have

$$
\begin{aligned}
\left\|\mathcal{F}_{\mathrm{n}, \theta}(\cdot)-\mathcal{F}(\cdot)\right\|_{\mathcal{L}^{2}(\Omega)} & \leq \sup _{a_{p}>0}\left(a_{p}^{2-\kappa} \frac{\mathrm{n}}{\mathcal{Q}_{0}^{2} \mathcal{M}^{2}+a_{p}^{2} \mathrm{n}}\right) \mathcal{P} \\
& \leq \begin{cases}\mathcal{N}_{1}\left(\kappa, \mathcal{M}^{2}\right) \mathrm{n} \frac{\kappa}{2} \mathcal{P}, & 0<\kappa<2, \\
\mathcal{N}_{2}\left(\kappa, \mathcal{M}^{2}\right) \mathrm{n} \mathcal{P}, & \kappa \geq 2 .\end{cases}
\end{aligned}
$$

Substituting the above inequality into (24) and applying (25), we get

$$
\left\|\mathcal{F}_{\mathrm{n}, \theta}^{\varepsilon}(\cdot)-\mathcal{F}(\cdot)\right\|_{\mathcal{L}^{2}(\Omega)} \leq C_{\theta}^{\frac{1}{2}} \mathrm{n}^{-\frac{1}{2}} \varepsilon+ \begin{cases}\mathcal{N}_{1}\left(\kappa, \mathcal{M}^{2}\right) \mathrm{n}^{\frac{\kappa}{2}} \mathcal{P}, & 0<\kappa<2, \\ \mathcal{N}_{2}\left(\kappa, \mathcal{M}^{2}\right) \mathrm{n} \mathcal{P}, & \kappa \geq 2\end{cases}
$$

Choose the regularization parameter $\mathrm{n}$ by

$$
\mathrm{n}= \begin{cases}\left(\frac{\varepsilon}{\mathcal{P}}\right)^{\frac{2}{\kappa+1}}, & 0<\kappa<2 \\ \left(\frac{\varepsilon}{\mathcal{P}}\right)^{\frac{2}{3}}, & \kappa \geq 2\end{cases}
$$


then we have

$$
\left\|\mathscr{F}_{\mathrm{n}, \theta}^{\varepsilon}(\cdot)-\mathcal{F}(\cdot)\right\|_{\mathcal{L}^{2}(\Omega)} \leq \begin{cases}\left(C_{\theta}^{\frac{1}{2}}+\mathcal{N}_{1}\left(\kappa, \mathcal{M}^{2}\right)\right) \mathcal{P}^{\frac{1}{\kappa+1}} \varepsilon^{\frac{\kappa}{\kappa+1}}, & 0<\kappa<2, \\ \left(C_{\theta}^{\frac{1}{2}}+\mathcal{N}_{2}\left(\kappa, \mathcal{M}^{2}\right)\right) \mathcal{P}^{\frac{1}{3}} \varepsilon^{\frac{2}{3}}, & \kappa \geq 2 .\end{cases}
$$

The proof is completed.

\subsection{A posteriori parameter choice rule and convergence estimate}

Now, based on Morozov's discrepancy principle [9], we consider the choice of an a posteriori regularization. Let us choose the regularization parameter $\mathrm{n}$ such that

$$
\left\|\mathcal{K} \mathcal{F}_{\mathrm{n}, \theta}^{\varepsilon}-\Theta^{\varepsilon}\right\|_{\mathcal{L}^{2}(\Omega)}=\chi \varepsilon,
$$

where $\left\|\Theta^{\varepsilon}\right\|_{\mathcal{L}^{2}(\Omega)} \geq \chi \varepsilon>0$. Choose $\chi>1$, and the bound for $\mathrm{n}$ is given and depends on $\varepsilon$ and $\mathcal{P}$.

Lemma 4.2 If $n$ satisfies (27), we can get the following inequality:

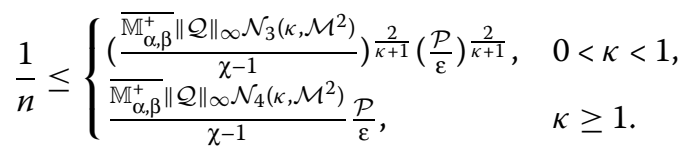

Proof From (27), we have

$$
\begin{aligned}
\chi \varepsilon= & \left\|\mathcal{K} \mathcal{F}_{\mathrm{n}, \theta}^{\varepsilon}-\Theta^{\varepsilon}\right\|_{\mathcal{L}^{2}(\Omega)} \\
\leq & \| \sum_{p=1}^{\infty}\left(1-\frac{\left(\frac{1}{\beta^{\alpha}} \int_{0}^{T}\left(T^{\beta}-\tau^{\beta}\right)^{\alpha-1} \boldsymbol{E}\left(-\frac{a_{p}}{\beta^{\alpha}}\left(T^{\beta}-\tau^{\beta}\right)^{\alpha}\right) \mathcal{Q}(\tau) d\left(\tau^{\beta}\right)\right)^{2 \theta}}{\left[\left(\frac{1}{\beta^{\alpha}} \int_{0}^{T}\left(T^{\beta}-\tau^{\beta}\right)^{\alpha-1} \boldsymbol{E}\left(-\frac{a_{p}}{\beta^{\alpha}}\left(T^{\beta}-\tau^{\beta}\right)^{\alpha}\right) \mathcal{Q}(\tau) d\left(\tau^{\beta}\right)\right)^{2}+\mathrm{n}\right]^{\theta}}\right) \\
& \times\left\langle\Theta^{\varepsilon}, w_{p}\right\rangle w_{p} \|_{\mathcal{L}^{2}(\Omega)} \\
\leq & \left\|\Theta^{\varepsilon}-\Theta\right\|_{\mathcal{L}^{2}(\Omega)}+\| \sum_{p=1}^{\infty}\left[\frac{\mathrm{n}}{\left(\frac{1}{\beta^{\alpha}} \int_{0}^{T}\left(T^{\beta}-\tau^{\beta}\right)^{\alpha-1} \boldsymbol{E}\left(-\frac{a_{p}}{\beta^{\alpha}}\left(T^{\beta}-\tau^{\beta}\right)^{\alpha}\right) \mathcal{Q}(\tau) d\left(\tau^{\beta}\right)\right)^{2}+\mathrm{n}}\right]^{\theta} \\
& \times\left\langle\Theta, w_{p}\right\rangle w_{p} \|_{\mathcal{L}^{2}(\Omega)} \\
= & \varepsilon+\left\|\sum_{p=1}^{\infty}\left[\frac{\mathrm{n}\left(\frac{1}{\beta^{\alpha}} \int_{0}^{T}\left(T^{\beta}-\tau^{\beta}\right)^{\alpha-1} \boldsymbol{E}\left(-\frac{a_{p}}{\beta^{\alpha}}\left(T^{\beta}-\tau^{\beta}\right)^{\alpha}\right) \mathcal{Q}(\tau) d\left(\tau^{\beta}\right)\right)}{\left(\frac{1}{\beta^{\alpha}} \int_{0}^{T}\left(T^{\beta}-\tau^{\beta}\right)^{\alpha-1} \boldsymbol{E}\left(-\frac{a_{p}}{\beta^{\alpha}}\left(T^{\beta}-\tau^{\beta}\right)^{\alpha}\right) \mathcal{Q}(\tau) d\left(\tau^{\beta}\right)\right)^{2}+\mathrm{n}}\right]^{\theta}\left\langle\mathcal{F}, w_{p}\right\rangle w_{p}\right\|_{\mathcal{L}^{2}(\Omega)} \\
:= & \varepsilon+\mathcal{A}_{1} .
\end{aligned}
$$


In view of Lemma 2.6 and $\theta \in\left(\frac{1}{2}, 1\right]$, it implies that

$$
\begin{aligned}
\mathcal{A}_{1} & =\left\|\sum_{p=1}^{\infty}\left[\frac{\mathrm{n} \frac{1}{a_{p}} \overline{\mathbb{M}_{\alpha, \beta}^{+}}\|\mathcal{Q}\|_{\infty}}{\left(\frac{1}{a_{p}} \mathcal{Q}_{0} \mathcal{M}\right)^{2}+\mathrm{n}}\right]^{\theta}\left\langle\mathcal{F}, w_{p}\right\rangle w_{p}\right\|_{\mathcal{L}^{2}(\Omega)} \\
& \leq\left(\sum_{p=1}^{\infty}\left[\frac{\mathrm{n} a_{p}^{1-\kappa} \overline{\mathbb{M}_{\alpha, \beta}^{+}}\|\mathcal{Q}\|_{\infty}}{\mathcal{Q}_{0}^{2} \mathcal{M}^{2}+a_{p}^{2} \mathrm{n}}\right]^{2} a_{p}^{2 \kappa}\left|\left\langle\mathcal{F}, w_{p}\right\rangle\right|^{2}\right)^{\frac{1}{2}} .
\end{aligned}
$$

By bound condition (12) and Lemma 2.5, we obtain

$$
\begin{aligned}
\mathcal{A}_{1} & \leq \sup _{a_{p}>0}\left[\frac{\mathrm{n} a_{p}^{1-\kappa} \overline{\mathbb{M}_{\alpha, \beta}^{+}}\|\mathcal{Q}\|_{\infty}}{\mathcal{Q}_{0}^{2} \mathcal{M}^{2}+a_{p}^{2} \mathrm{n}}\right]\|\mathcal{F}\|_{\mathcal{D}\left((-\mathcal{B})^{-\kappa}\right)} \\
& \leq \begin{cases}\overline{\mathbb{M}_{\alpha, \beta}^{+}}\|\mathcal{Q}\|_{\infty} \mathcal{N}_{3}\left(\kappa, \mathcal{M}^{2}\right) \mathrm{n}{ }^{\frac{1+\kappa}{2}} \mathcal{P}, & 0<\kappa<1, \\
\overline{\mathbb{M}_{\alpha, \beta}^{+}}\|\mathcal{Q}\|_{\infty} \mathcal{N}_{4}\left(\kappa, \mathcal{M}^{2}\right) \mathrm{n} \mathcal{P}, & \kappa \geq 1 .\end{cases}
\end{aligned}
$$

Combining the above equations, we deduce that

$$
(\chi-1) \varepsilon \leq \begin{cases}\overline{\mathbb{M}_{\alpha, \beta}^{+}}\|\mathcal{Q}\|_{\infty} \mathcal{N}_{3}\left(\kappa, \mathcal{M}^{2}\right) \mathrm{n}^{\frac{1+\kappa}{2}} \mathcal{P}, & 0<\kappa<1 \\ \overline{\mathbb{M}_{\alpha, \beta}^{+}}\|\mathcal{Q}\|_{\infty} \mathcal{N}_{4}\left(\kappa, \mathcal{M}^{2}\right) \mathrm{n} \mathcal{P}, & \kappa \geq 1\end{cases}
$$

This yields

$$
\frac{1}{\mathrm{n}} \leq \begin{cases}\left.\frac{\left(\overline{\mathbb{M}_{\alpha, \beta}^{+}} \| \mathcal{Q}_{\infty} \mathcal{N}_{3}\left(\kappa, \mathcal{M}^{2}\right)\right.}{\chi-1}\right) \frac{2}{\kappa+1}\left(\frac{\mathcal{P}}{\varepsilon}\right)^{\frac{2}{\kappa+1}}, & 0<\kappa<1 \\ \frac{\overline{\mathbb{M}_{\alpha, \beta}^{+}}\|\mathcal{Q}\|_{\infty} \mathcal{N}_{4}\left(\kappa, \mathcal{M}^{2}\right)}{\chi-1} \frac{\mathcal{P}}{\varepsilon}, & \kappa \geq 1\end{cases}
$$

Theorem 4.2 We recall that $n$ in Lemma 4.2 and bound condition (12) hold. Then we have

$$
\begin{aligned}
& \left\|\mathcal{F}_{n, \theta}^{\varepsilon}(\cdot)-\mathcal{F}(\cdot)\right\|_{\mathcal{L}^{2}(\Omega)} \\
& \quad \leq\left(\frac{\chi+1}{\mathcal{Q}_{0} \mathcal{M}}\right)^{\frac{\kappa}{\kappa+1}} \mathcal{P}^{\frac{1}{\kappa+1}} \varepsilon^{\frac{\kappa}{\kappa+1}}+ \begin{cases}C_{\theta}^{\frac{1}{2}}\left(\frac{\overline{\mathbb{M}_{\alpha, \beta}^{+}}\|\mathcal{Q}\|_{\infty} \mathcal{N}_{3}\left(\kappa, \mathcal{M}^{2}\right)}{\chi-1}\right)^{\frac{2}{\kappa+1}} \mathcal{P}^{\frac{1}{\kappa+1}} \varepsilon^{\frac{\kappa}{\kappa+1}}, & 0<\kappa<1, \\
C_{\theta}^{\frac{1}{2}}\left(\frac{\overline{\mathbb{M}_{\alpha, \beta}^{+}}\|\mathcal{Q}\|_{\infty} \mathcal{N}_{4}\left(\kappa, \mathcal{M}^{2}\right)}{\chi-1}\right)^{\frac{1}{2}} \mathcal{P}^{\frac{1}{2}} \varepsilon^{\frac{1}{2}}, & \kappa \geq 1 .\end{cases}
\end{aligned}
$$

Proof From the triangle inequality, we obtain

$$
\left\|\mathcal{F}_{\mathrm{n}, \theta}^{\varepsilon}(\cdot)-\mathcal{F}(\cdot)\right\|_{\mathcal{L}^{2}(\Omega)} \leq\left\|\mathcal{F}_{\mathrm{n}, \theta}^{\varepsilon}(\cdot)-\mathcal{F}_{\mathrm{n}, \theta}(\cdot)\right\|_{\mathcal{L}^{2}(\Omega)}+\left\|\mathcal{F}_{\mathrm{n}, \theta}(\cdot)-\mathcal{F}(\cdot)\right\|_{\mathcal{L}^{2}(\Omega)}
$$

First, the estimate for $\left\|\mathcal{F}_{\mathrm{n}, \theta}^{\varepsilon}(\cdot)-\mathcal{F}_{\mathrm{n}, \theta}(\cdot)\right\|_{\mathcal{L}^{2}(\Omega)}$ is given. By (25), we get

$$
\left\|\mathcal{F}_{\mathrm{n}, \theta}^{\varepsilon}(\cdot)-\mathcal{F}_{\mathrm{n}, \theta}(\cdot)\right\|_{\mathcal{L}^{2}(\Omega)} \leq C_{\theta}^{\frac{1}{2}} \mathrm{n}^{-\frac{1}{2}} \varepsilon
$$

Substituting (28) into the above equation, we get

$$
\left\|\mathcal{F}_{\mathrm{n}, \theta}^{\varepsilon}(\cdot)-\mathcal{F}_{\mathrm{n}, \theta}(\cdot)\right\|_{\mathcal{L}^{2}(\Omega)} \leq \begin{cases}C_{\theta}^{\frac{1}{2}}\left(\frac{\overline{\mathbb{M}_{\alpha, \beta}^{+}} \| \mathcal{Q}_{\infty} \mathcal{N}_{3}\left(\kappa, \mathcal{M}^{2}\right)}{\chi \chi^{\prime}-1}\right)^{\frac{2}{\kappa+1}} \mathcal{P}^{\frac{1}{\kappa+1}} \varepsilon^{\frac{\kappa}{\kappa+1}}, & 0<\kappa<1, \\ C_{\theta}^{\frac{1}{2}}\left(\frac{\overline{\mathbb{M}_{\alpha, \beta}^{+} \|}\|\|_{\infty} \mathcal{N}_{4}\left(\kappa, \mathcal{M}^{2}\right)}{\chi-1}\right)^{\frac{1}{2}} \mathcal{P}^{\frac{1}{2}} \varepsilon^{\frac{1}{2}}, & \kappa \geq 1 .\end{cases}
$$


In the next step, we prove the bound for the second term. Similar to the above equation, we have

$$
\begin{aligned}
& \left\|\mathcal{F}_{\mathrm{n}, \theta}(\cdot)-\mathcal{F}(\cdot)\right\|_{\mathcal{L}^{2}(\Omega)} \\
& \quad \leq\left\|\sum_{p=1}^{\infty} \frac{\mathrm{n}^{\theta}\left(\frac{1}{\beta^{\alpha}} \int_{0}^{T}\left(T^{\beta}-\tau^{\beta}\right)^{\alpha-1} \boldsymbol{E}\left(-\frac{a_{p}}{\beta^{\alpha}}\left(T^{\beta}-\tau^{\beta}\right)^{\alpha}\right) \mathcal{Q}(\tau) d\left(\tau^{\beta}\right)\right)^{-1}}{\left[\left(\frac{1}{\beta^{\alpha}} \int_{0}^{T}\left(T^{\beta}-\tau^{\beta}\right)^{\alpha-1} \boldsymbol{E}\left(-\frac{a_{p}}{\beta^{\alpha}}\left(T^{\beta}-\tau^{\beta}\right)^{\alpha}\right) \mathcal{Q}(\tau) d(\tau)\right)^{2}+\mathrm{n}\right]^{\Theta}} \Theta_{p}\right\|_{\mathcal{L}^{2}(\Omega)} .
\end{aligned}
$$

Applying Hölder's inequality, we have

$$
\begin{aligned}
& \left\|\mathcal{F}_{\mathrm{n}, \theta}(\cdot)-\mathcal{F}(\cdot)\right\|_{\mathcal{L}^{2}(\Omega)} \\
& \quad \leq \mathcal{A}_{2}\left\|\sum_{p=1}^{\infty} \frac{\mathrm{n}^{\theta}\left(\frac{1}{\beta^{\alpha}} \int_{0}^{T}\left(T^{\beta}-\tau^{\beta}\right)^{\alpha-1} \boldsymbol{E}\left(-\frac{a_{p}}{\beta^{\alpha}}\left(T^{\beta}-\tau^{\beta}\right)^{\alpha}\right) \mathcal{Q}(\tau) d\left(\tau^{\beta}\right)\right)^{-1}}{\left[\left(\frac{1}{\beta^{\alpha}} \int_{0}^{T}\left(T^{\beta}-\tau^{\beta}\right)^{\alpha-1} \boldsymbol{E}\left(-\frac{a_{p}}{\beta^{\alpha}}\left(T^{\beta}-\tau^{\beta}\right)^{\alpha}\right) \mathcal{Q}(\tau) d\left(\tau^{\beta}\right)\right)^{2}+\mathrm{n}\right]^{\theta}} \Theta_{p}\right\|_{\mathcal{L}^{2}(\Omega)}^{\frac{\kappa}{\kappa+1}},
\end{aligned}
$$

where

$$
\mathcal{A}_{2}=\left\|\sum_{p=1}^{\infty}\left[\frac{\mathrm{n}}{\left(\frac{1}{\beta^{\alpha}} \int_{0}^{T}\left(T^{\beta}-\tau^{\beta}\right)^{\alpha-1} \boldsymbol{E}\left(-\frac{a_{p}}{\beta^{\alpha}}\left(T^{\beta}-\tau^{\beta}\right)^{\alpha}\right) \mathcal{Q}(\tau) d\left(\tau^{\beta}\right)\right)^{2}+\mathrm{n}}\right]^{\theta} \mathcal{F}_{p}\right\|_{\mathcal{L}^{2}(\Omega)}^{\frac{1}{\kappa^{\alpha+1}}} .
$$

By bound condition (12), we get

$$
\mathcal{A}_{2} \leq\left(\sum_{p=1}^{\infty} a_{p}^{-2 \kappa} a_{p}^{2 \kappa}\left|\mathcal{F}_{p}\right|^{2}\right)^{\frac{1}{2(\kappa+1)}} \leq \sup _{a_{p}>0}\left(a_{p}\right)^{-\frac{\kappa}{\kappa+1}} \mathcal{P}^{\frac{1}{\kappa+1}} .
$$

Hence, we deduce that

$$
\begin{aligned}
& \left\|\mathcal{F}_{\mathrm{n}, \theta}(\cdot)-\mathcal{F}(\cdot)\right\|_{\mathcal{L}^{2}(\Omega)} \\
& \leq \sup _{a_{p}>0}\left(a_{p} \frac{1}{\beta^{\alpha}} \int_{0}^{T}\left(T^{\beta}-\tau^{\beta}\right)^{\alpha-1} \boldsymbol{E}\left(-\frac{a_{p}}{\beta^{\alpha}}\left(T^{\beta}-\tau^{\beta}\right)^{\alpha}\right) \mathcal{Q}(\tau) d\left(\tau^{\beta}\right)\right)^{-\frac{\kappa}{\kappa+1}} \mathcal{P}^{\frac{1}{\kappa+1}} \\
& \quad \times\left(\left\|\Theta^{\varepsilon}-\Theta\right\|_{\mathcal{L}^{2}(\Omega)}\right. \\
& \left.\quad+\left\|\sum_{p=1}^{\infty} \frac{\mathrm{n}^{\theta}}{\left[\left(\frac{1}{\beta^{\alpha}} \int_{0}^{T}\left(T^{\beta}-\tau^{\beta}\right)^{\alpha-1} \boldsymbol{E}\left(-\frac{a_{p}}{\beta^{\alpha}}\left(T^{\beta}-\tau^{\beta}\right)^{\alpha}\right) \mathcal{Q}(\tau) d\left(\tau^{\beta}\right)\right)^{2}+\mathrm{n}\right]^{\theta}} \Theta_{p}^{\varepsilon}\right\|_{\mathcal{L}^{2}(\Omega)}\right)^{\frac{\kappa}{\kappa+1}} .
\end{aligned}
$$

In view of (4), (17), and Lemma (2.6), we have that

$$
\left\|\mathcal{F}_{\mathrm{n}, \theta}(\cdot)-\mathcal{F}(\cdot)\right\|_{\mathcal{L}^{2}(\Omega)} \leq\left(\frac{\chi+1}{\mathcal{Q}_{0} \mathcal{M}}\right)^{\frac{\kappa}{\kappa+1}} \mathcal{P}^{\frac{1}{\kappa+1}} \mathcal{\varepsilon}^{\frac{\kappa}{\kappa+1}} .
$$

From the above, we can conclude that

$$
\begin{aligned}
& \left\|\mathcal{F}_{\mathrm{n}, \theta}^{\varepsilon}(\cdot)-\mathcal{F}(\cdot)\right\|_{\mathcal{L}^{2}(\Omega)} \\
& \quad \leq\left(\frac{\chi+1}{\mathcal{Q}_{0} \mathcal{M}}\right)^{\frac{\kappa}{\kappa+1}} \mathcal{P}^{\frac{1}{\kappa+1}} \varepsilon^{\frac{\kappa}{\kappa+1}}+ \begin{cases}C_{\theta}^{\frac{1}{2}}\left(\frac{\overline{\mathbb{M}_{\alpha, \beta}^{+}} \| \mathcal{Q}_{\infty} \mathcal{N}_{3}\left(\kappa, \mathcal{M}^{2}\right)}{\chi-1}\right)^{\frac{2}{\kappa+1}} \mathcal{P}^{\frac{1}{\kappa+1}} \varepsilon^{\frac{\kappa}{\kappa+1}}, & 0<\kappa<1, \\
C_{\theta}^{\frac{1}{2}}\left(\frac{\overline{\mathbb{M}_{\alpha, \beta}^{+}}\|\|_{\infty} \mathcal{N}_{4}\left(\kappa, \mathcal{M}^{2}\right)}{\chi-1}\right)^{\frac{1}{2}} \mathcal{P}^{\frac{1}{2}} \varepsilon^{\frac{1}{2}}, & \kappa \geq 1 .\end{cases}
\end{aligned}
$$

This ends the proof. 


\section{Numerical example}

In this section, we present a numerical example to illustrate the proposed method.

Step 1: Set up some essentials for example. Let $\alpha, \beta \in(0,1), T=1$ be a fixed value, $\boldsymbol{\Omega}=$ $[0, \pi]$ and $\mathcal{B}=-\Delta$ on $\mathcal{L}^{2}(0, \pi)$. Then we have the eigenvalues $a_{p}=p^{2}, p=1,2, \ldots$, and the corresponding eigenfunction $w_{p}(\mathrm{x})=\sqrt{\frac{2}{\pi}} \sin (p \mathrm{x})$.

To perform the calculation in this example, we use the Matlab software, we use the code made by JC Medina [19] to compute the integral via Simpson's rule in the interval $[a, b]$, and the Mittag-Leffler function by Igor Podlubny [22].

Next, we choose the exact data function $\mathrm{g}, \mathcal{F}$, and $\mathcal{Q}$ as follows:

$$
\mathrm{g}(\mathrm{x})=\sqrt{\frac{2}{\pi}} \sin (\mathrm{x}), \quad \mathcal{F}(\mathrm{x})=\sqrt{\frac{2}{\pi}} \sin (3 \mathrm{x}), \quad \mathcal{Q}(t)=\frac{\left(1-t^{\beta}\right)^{1-\alpha}}{\boldsymbol{E}\left(\frac{-9\left(1-t^{\beta}\right)^{\alpha}}{\beta^{\alpha}}\right)} .
$$

Step 2: Partitioning of axes: Let $N_{\mathrm{x}}, N_{t}$ be given positive integers, a uniform Cartesian grid is given by

$$
\mathrm{x}_{i}=\frac{\pi(i-1)}{N_{\mathrm{x}}}, \quad t_{j}=\frac{j-1}{N_{t}}, \quad \text { where } i=1,2, \ldots, N_{\mathrm{x}}+1 \text {, and } j=1,2, \ldots, N_{t}+1 .
$$

Step 3: Model of noise: From (9) and (33), the value of the final time is given by

$$
\mathscr{H}(\mathrm{x})=\sqrt{\frac{2}{\pi}}\left[E_{\alpha}\left(-\frac{1}{\beta^{\alpha}}\right) \sin (\mathrm{x})+\frac{1}{\beta^{\alpha}} \sin (3 x)\right] .
$$

Then we consider the noise model satisfying (see an example in Fig. 1)

$$
\mathscr{H}^{\varepsilon}=\mathscr{H}+\varepsilon \operatorname{randn}(\mathscr{H}(\cdot)),
$$

where the noise level $\varepsilon \longrightarrow 0^{+}$and the function $\operatorname{randn}(\cdot)$ generates arrays of random numbers whose elements are normally distributed.

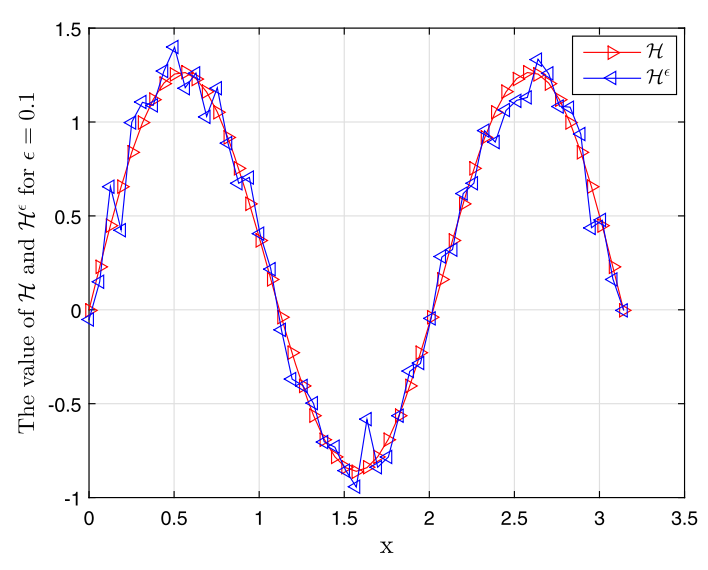

Figure 1 The function $\mathscr{H}$ and $\mathscr{H}^{\varepsilon}$ for $\mathrm{x} \in[0, \pi], \alpha=\beta=0.5$, and $\boldsymbol{\varepsilon}=0.1$ 
Step 4: The regularization results:

Case 1: Applying the fractional Landweber regularization method, we have the following solution with the observation data $\mathscr{H}^{\varepsilon}$ :

$$
\begin{aligned}
& \mathcal{F}_{\mathrm{m}, \boldsymbol{\theta}}^{1, \mathcal{\varepsilon}}(\mathrm{x}) \\
& =\sum_{p=1}^{N(p)} \frac{\left[1-\left(1-c\left[\frac{1}{\beta^{\alpha}} \int_{0}^{1}\left(1-\tau^{\beta}\right)^{\alpha-1} \boldsymbol{E}\left(-\frac{a_{p}}{\beta^{\alpha}}\left(1-\tau^{\beta}\right)^{\alpha}\right) \mathcal{Q}(\tau) d\left(\tau^{\beta}\right)\right]^{2}\right)^{\mathrm{m}}\right]^{\Theta}}{\frac{1}{\beta^{\alpha}} \int_{0}^{1}\left(1-\tau^{\beta}\right)^{\alpha-1} \boldsymbol{E}\left(-\frac{a_{p}}{\beta^{\alpha}}\left(1-\tau^{\beta}\right)^{\alpha}\right) \mathcal{Q}(\tau) d\left(\tau^{\beta}\right)}\left\langle\Theta^{\varepsilon}, w_{p}\right| w_{p},
\end{aligned}
$$

here $\Theta:=\mathscr{H}-\sum_{p=1}^{N(p)}\left(E_{\alpha, 1}\left(-\frac{a_{p}}{\beta^{\alpha}}\right) \mathrm{g}_{p}\right) w_{p}(\mathrm{x})$, and $\Theta^{\varepsilon}:=\mathscr{H}^{\varepsilon}-\sum_{p=1}^{N(p)}\left(E_{\alpha, 1}\left(-\frac{a_{p}}{\beta^{\alpha}}\right) \mathrm{g}_{p}\right) w_{p}(\mathrm{x}), \theta \in$ $\left(\frac{1}{2}, 1\right]$ is the fractional order, $\mathrm{m}>0$ is the iterative step, and $N(p)$ is a truncation parameter of Fourier series.

Case 2: Applying the fractional Tikhonov regularization method, we have the following solution with the observation data $\mathscr{H}^{\varepsilon}$ :

$$
\begin{aligned}
& \mathcal{F}_{\mathrm{n}, \theta}^{2, \varepsilon}(\mathrm{x}) \\
& =\sum_{p=1}^{N(p)} \frac{\left(\frac{1}{\beta^{\alpha}} \int_{0}^{1}\left(1-\tau^{\beta}\right)^{\alpha-1} \boldsymbol{E}\left(-\frac{a_{p}}{\beta^{\alpha}}\left(1-\tau^{\beta}\right)^{\alpha}\right) \mathcal{Q}(\tau) d\left(\tau^{\beta}\right)\right)^{2 \theta-1}}{\left[\left(\frac{1}{\beta^{\alpha}} \int_{0}^{1}\left(1-\tau^{\beta}\right)^{\alpha-1} \boldsymbol{E}\left(-\frac{a_{p}}{\beta^{\alpha}}\left(1-\tau^{\beta}\right)^{\alpha}\right) \mathcal{Q}(\tau) d\left(\tau^{\beta}\right)\right)^{2}+\mathrm{n}\right]^{\theta}}\left\langle\Theta^{\varepsilon}, w_{p}\right) w_{p},
\end{aligned}
$$

where the regularization parameter $\mathrm{n}>0$, and $\theta \in\left(\frac{1}{2}, 1\right]$ is the fractional order.

The absolute error estimation Error between the exact source function and the regularized source function in two cases is as follows:

$$
\begin{aligned}
& \operatorname{Error}_{\mathrm{m}}^{1}(\alpha, \beta)=\sqrt{\frac{\sum_{i=1}^{N_{\mathrm{x}}+1}\left|\mathcal{F}_{\mathrm{m}, \theta}^{1, \varepsilon}\left(\mathrm{x}_{i}\right)-\mathcal{F}\left(\mathrm{x}_{i}\right)\right|^{2}}{N_{\mathrm{x}}+1}}, \\
& \operatorname{Error}_{\mathrm{n}}^{2}(\alpha, \beta)=\sqrt{\frac{\sum_{i=1}^{N_{\mathrm{x}}+1}\left|\mathcal{F}_{\mathrm{n}, \theta}^{2, \varepsilon}\left(\mathrm{x}_{i}\right)-\mathcal{F}\left(\mathrm{x}_{i}\right)\right|^{2}}{N_{\mathrm{x}}+1}} .
\end{aligned}
$$

The results of this section are presented in Table 1, Figs. 2, 3, and 4. In Table 1, we present the error estimation between the exact and regularized source functions. We also show

\begin{tabular}{|c|c|c|c|c|}
\hline \multirow[t]{2}{*}{$\operatorname{Error}_{m, n}^{1,2}(\alpha, \beta)$} & \multicolumn{4}{|c|}{$N_{\mathrm{x}}=40, N(p)=10, \mathrm{~m}=\mathrm{n}=5, c=0.1, \theta=0.3$} \\
\hline & $\overline{\varepsilon:}$ & 0.1 & 0.01 & 0.001 \\
\hline $\begin{array}{l}\text { Error }_{m}^{1}(0.1,0.1) \\
\text { Error }_{n}^{2}(0.1,0.1)\end{array}$ & & $\begin{array}{l}4.622039876238438 \\
6.865541133036323\end{array}$ & $\begin{array}{l}0.813894458788424 \\
1.307095435681400\end{array}$ & $\begin{array}{l}0.034641254935990 \\
0.080884577605506\end{array}$ \\
\hline $\begin{array}{l}\text { Error }_{m}^{1}(0.5,0.5) \\
\text { Error }_{n}^{2}(0.5,0.5)\end{array}$ & & $\begin{array}{l}3.612281616966185 \\
5.675784647354515\end{array}$ & $\begin{array}{l}0.661448882414577 \\
1.121826494146178\end{array}$ & $\begin{array}{l}0.036340894224658 \\
0.079463345903329\end{array}$ \\
\hline $\begin{array}{l}\operatorname{Error}_{m}^{1}(0.9,0.9) \\
\operatorname{Error}_{n}^{2}(0.9,0.9)\end{array}$ & & $\begin{array}{l}4.499633069709045 \\
4.446871112063462\end{array}$ & $\begin{array}{l}0.994936684930523 \\
1.090617580396543\end{array}$ & $\begin{array}{l}0.043721289744985 \\
0.013012608028477\end{array}$ \\
\hline
\end{tabular}
the graph of the source functions for $\alpha=\beta=0.9$ and $\varepsilon \in\{0.1,0.01,0.001\}$, respectively. From the error table and the figures above, we can see that the smaller the $\varepsilon$, the better the computed approximation. In particular, the regularized source function approaches exact source function as $\varepsilon$ tends to zero.

Table 1 The error estimation between the exact and regularized source functions for $\mathrm{x} \in[0, \pi]$ 


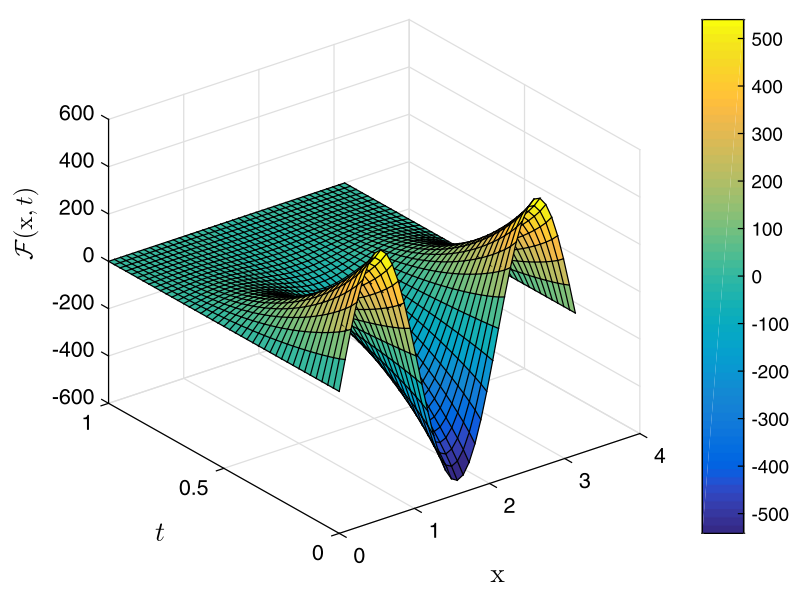

(a) $\mathcal{F}(\mathrm{x}, t)$ for $(\mathrm{x}, t) \in(0, \pi) \times(0,1)$

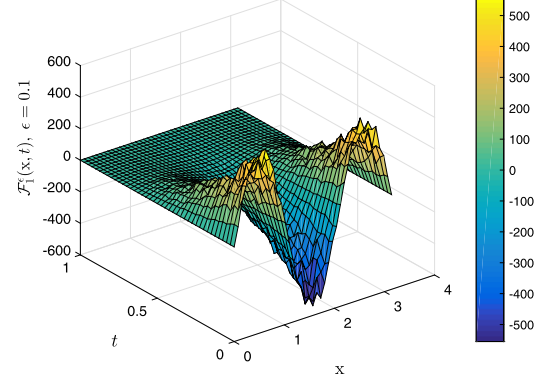

(b) $\mathcal{F}_{1}^{\epsilon}(\mathrm{x}, t)$ for $(\mathrm{x}, t) \in(0, \pi) \times(0,1)$

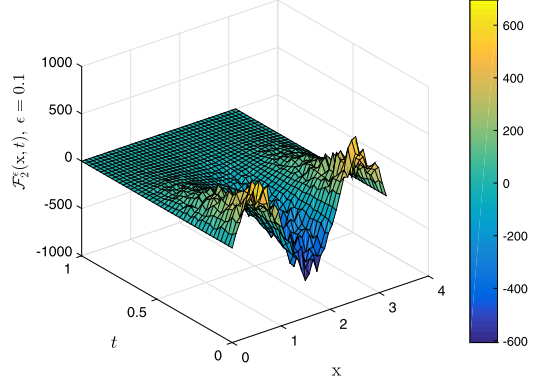

(c) $\mathcal{F}_{2}^{\epsilon}(\mathrm{x}, t)$ for $(\mathrm{x}, t) \in(0, \pi) \times(0,1)$

Figure 2 The source functions $\mathcal{F}(\mathrm{x}, t)=\mathscr{F}(\mathrm{x}) \mathcal{Q}(t), \mathcal{F}_{1}^{\mathcal{\varepsilon}}(\mathrm{x}, t)=\mathscr{F}_{\mathrm{m}, \boldsymbol{\theta}}^{1, \varepsilon}(\mathrm{x}) \mathcal{Q}(t)$, and $\mathcal{F}_{2}^{\mathcal{\varepsilon}}(\mathrm{x}, t)=\mathscr{F}_{\mathrm{n}, \boldsymbol{\theta}}^{2, \mathcal{\varepsilon}}(\mathrm{x}) \mathcal{Q}(t)$ for $(\mathrm{x}, t) \in(0, \pi) \times(0,1), \varepsilon=0.1, \alpha=\beta=0.9$

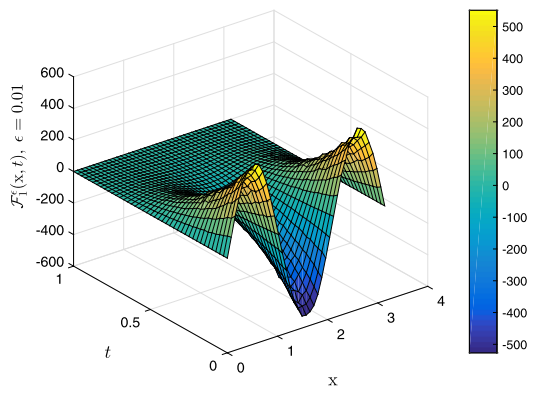

(a) $\mathcal{F}_{1}^{\epsilon}(\mathrm{x}, t)$ for $(\mathrm{x}, t) \in(0, \pi) \times(0,1)$

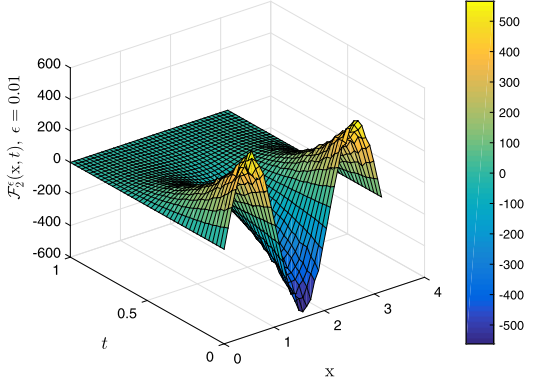

(b) $\mathcal{F}_{2}^{\epsilon}(\mathrm{x}, t)$ for $(\mathrm{x}, t) \in(0, \pi) \times(0,1)$

Figure 3 The source functions $\mathcal{F}_{1}^{\varepsilon}(\mathrm{x}, t)=\mathcal{F}_{\mathrm{m}, \boldsymbol{\theta}}^{1, \varepsilon}(\mathrm{x}) \mathcal{Q}(t)$ and $\mathcal{F}_{2}^{\varepsilon}(\mathrm{x}, t)=\mathcal{F}_{\mathrm{n}, \boldsymbol{\theta}}^{2, \varepsilon}(\mathrm{x}) \mathcal{Q}(t)$ for $(\mathrm{x}, t) \in(0, \pi) \times(0,1)$, $\varepsilon=0.01, \alpha=\beta=0.9$

\section{Conclusion}

The paper considers the regularization problem for the time-fractional diffusion equation with the hyper-Bessel operator. Firstly, through an example, we proved that the backward 


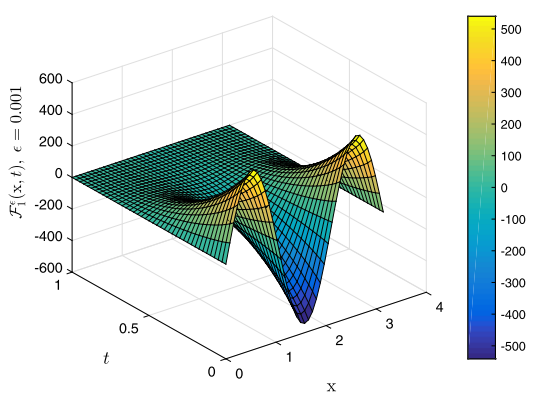

(a) $\mathcal{F}_{1}^{\epsilon}(\mathrm{x}, t)$ for $(\mathrm{x}, t) \in(0, \pi) \times(0,1)$

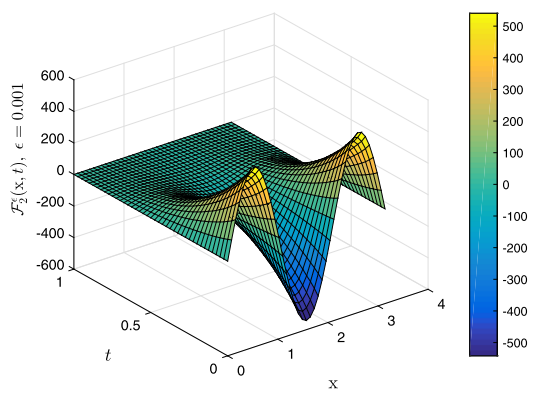

(b) $\mathcal{F}_{2}^{\epsilon}(\mathrm{x}, t)$ for $(\mathrm{x}, t) \in(0, \pi) \times(0,1)$

Figure 4 The source functions $\mathcal{F}_{1}^{\varepsilon}(\mathrm{x}, t)=\mathcal{F}_{\mathrm{m}, \boldsymbol{\theta}}^{1, \varepsilon}(\mathrm{x}) \mathcal{Q}(t)$ and $\mathcal{F}_{2}^{\varepsilon}(\mathrm{x}, t)=\mathcal{F}_{\mathrm{n}, \theta}^{2, \varepsilon}(\mathrm{x}) \mathcal{Q}(t)$ for $(\mathrm{x}, t) \in(0, \pi) \times(0,1)$, $\varepsilon=0.001, \alpha=\beta=0.9$

problem is not well posed (in the sense of Hadamard). Secondly, by the fractional Landweber and Tikhonov methods, we showed the results of the convergence rates for the regularized solution to the exact solution by using a priori and a posteriori regularization parameter choice rules.

Acknowledgements

Not applicable.

Funding

Not applicable.

Availability of data and materials

Not applicable.

\section{Competing interests}

The authors declare that they have no competing interests.

\section{Authors' contributions}

All authors contributed equally to the writing of this paper. The authors declare that the study was realized in collaboration with the same responsibility. All authors read and approved the final manuscript.

\section{Author details}

'Division of Applied Mathematics, Thu Dau Mot University, Binh Duong Province, Vietnam. ${ }^{2}$ Department of Mathematics and Computer Science, University of Science, Ho Chi Minh City, Vietnam. ${ }^{3}$ Vietnam National University, Ho Chi Minh City, Vietnam. ${ }^{4}$ Department of Mathematics, Cankaya University, Ankara, Turkey. ${ }^{5}$ Department of Medical Research, China Medical University Hospital, China Medical University, Taichung, Taiwan. ${ }^{6}$ Institute of Space Sciences,

Magurele-Bucharest, Romania. ${ }^{7}$ Applied Analysis Research Group, Faculty of Mathematics and Statistics, Ton Duc Thang University, Ho Chi Minh City, Vietnam.

\section{Publisher's Note}

Springer Nature remains neutral with regard to jurisdictional claims in published maps and institutional affiliations.

Received: 28 January 2020 Accepted: 20 May 2020 Published online: 01 June 2020

\section{References}

1. Agarwal, R.P., Lupulescu, V., O'Regan, D., ur Rahman, G.: Fractional calculus and fractional differential equations in nonreflexive Banach spaces. Commun. Nonlinear Sci. Numer. Simul. 20(1), 59-73 (2015)

2. Al-Musalhi, F., Al-Salti, N., Karimov, E.: Initial boundary value problems for a fractional differential equation with hyper-Bessel operator. Fract. Calc. Appl. Anal. 21(1), 200-219 (2018)

3. Baleanu, D., Diethelm, K., Scalas, E., Trujillo, J.J.: Fractional Calculus: Models and Numerical Methods. Series on Complexity, Nonlinearity and Chaos, vol. 3. World Scientific, Boston (2012)

4. Baleanu, D., Güvenç, Z.B., Machado, J.T. (eds.): New Trends in Nanotechnology and Fractional Calculus Applications. Springer, New York (2010)

5. Courant, R., Hilbert, D.: Methods of Mathematical Physics: Partial Differential Equations. Wiley, New York (2008)

6. Cuesta, E.: Some advances on image processing by means of fractional calculus. In: Nonlinear Science and Complexity, pp. 265-271. Springer, Dordrecht (2011) 
7. Diethelm, K.: The Analysis of Fractional Differential Equations: An Application-Oriented Exposition Using Differential Operators of Caputo Type. Springer, Berlin (2010)

8. Dimovski, I.: Operational calculus of a class of differential operators. C. R. Acad. Bulgare Sci. 19(12), 1111-1114 (1966)

9. Engl, H.W., Hanke, M., Neubauer, A.: Regularization of Inverse Problems. Mathematics and Its Applications, vol. 375. Springer, Berlin (1996)

10. Garra, R., Giusti, A., Mainardi, F., Pagnini, G.: Fractional relaxation with time-varying coefficient. Fract. Calc. Appl. Anal. $17(2), 424-439$ (2014)

11. Garra, R., Orsingher, E., Polito, F.: Fractional diffusion with time-varying coefficients. J. Math. Phys. 56, Article ID 093301 (2015)

12. Giona, M., Cerbelli, S., Roman, H.E.: Fractional diffusion equation and relaxation in complex viscoelastic materials. Phys. A, Stat. Mech. Appl. 191(1-4), 449-453 (1992)

13. Kilbas, A.A.A., Srivastava, H.M., Trujillo, J.J.: Theory and Applications of Fractional Differential Equations. Elsevier, Amsterdam (2006)

14. Kirsch, A.: An Introduction to the Mathematical Theory of Inverse Problem. Applied Mathematical Sciences, vol. 120. Springer, Berlin (2011)

15. Klann, E., Ramlau, R.: Regularization by fractional filter methods and data smoothing. Inverse Probl. 24(2), Article ID $025018(2008)$

16. Kutner, R.: Coherent spatio-temporal coupling in fractional wanderings. Renewed approach to continuous-time Lévy flights. In: Anomalous Diffusion from Basics to Applications, pp. 1-14. Springer, Berlin (1999)

17. Louis, A.K.: Inverse und Schlecht Gestellte Probleme. Springer, Berlin (2013)

18. Magin, R.L.: Fractional Calculus in Bioengineering. Begell House Publishers Inc., Redding (2006)

19. Medina, J.C.: Simpson's rule integration, MATLAB Central File Exchange. https://www.mathworks.com/matlabcentral/fileexchange/28726-simpson-s-rule-integration. Retrieved March 19, 2020

20. Metzler, R., Klafter, J.: The random walks guide to anomalous diffusion: a fractional dynamics approach. Phys. Rep. $339(1), 1-77(2000)$

21. Podlubny, l.: Fractional Differential Equations. Academic Press, San Diego (1999)

22. Podlubny, I.: MATLAB Central File Exchange. https://www.mathworks.com/matlabcentral/fileexchange/8738-mittag-leffler-function. Retrieved Apr 12, 2020

23. Samko, S.G., Killbas, A.A., Marichev, O.I.: Fractional Integrals and Derivatives-Theory and Applications. Gordon \& Breach, Linghorne (1993)

24. Scalas, E., Gorenflo, R., Mainardi, F.: Fractional calculus and continuous-time finance. Phys. A, Stat. Mech. Appl. 284(1-4), 376-384 (2000)

25. Tuan, N.H., Huynh, L.N., Baleanu, D., Can, N.H.: On a terminal value problem for a generalization of the fractional diffusion equation with hyper-Bessel operator. Math. Methods Appl. Sci. 43(6), 2858-2882 (2020)

26. Tuan, N.H., Huynh, L.N., Ngoc, T.B., Zhou, Y.: On a backward problem for nonlinear fractional diffusion equations. Appl. Math. Lett. 92, 76-84 (2019)

27. Vainikko, G.M., Veretennikov, A.Y.: Iteration Procedures in II-Posed Problems. Nauka, Moscow (1986) (in Russian)

28. Wang, J.G., Wei, T., Zhou, Y.B.: Tikhonov regularization method for a backward problem for the time-fractional diffusion equation. Appl. Math. Model. 37(18-19), 8518-8532 (2013)

29. Zhang, K.: Existence results for a generalization of the time-fractional diffusion equation with variable coefficients. Bound. Value Probl. 2019(1), Article ID 10 (2019)

30. Zhang, K.: Positive solution of nonlinear fractional differential equations with Caputo-like counterpart hyper-Bessel operators. Math. Methods Appl. Sci. 43(6), 2845-2857 (2020)

\section{Submit your manuscript to a SpringerOpen ${ }^{\circ}$ journal and benefit from:}

- Convenient online submission

- Rigorous peer review

- Open access: articles freely available online

- High visibility within the field

- Retaining the copyright to your article

Submit your next manuscript at $\boldsymbol{~ s p r i n g e r o p e n . c o m ~}$ 